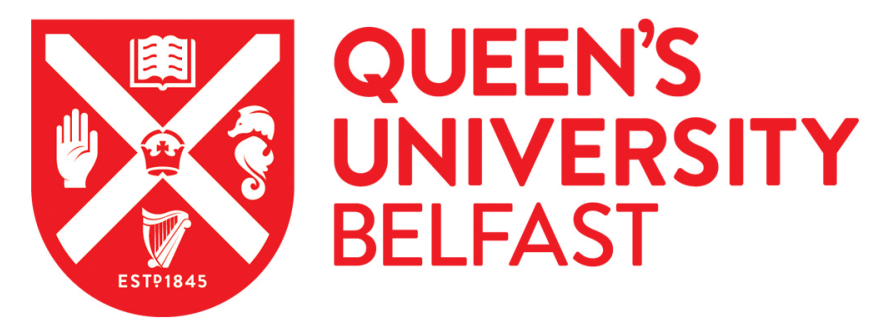

\title{
Influence of strain and polycrystalline ordering on magnetic properties of high moment rare earth metals and alloys
}

\begin{abstract}
Scheunert, G., Ward, C., Hendren, W. R., Lapicki, A. A., Hardeman, R., Mooney, M., Gubbins, M., \& Bowman, R. M. (2014). Influence of strain and polycrystalline ordering on magnetic properties of high moment rare earth metals and alloys. Journal of Physics D: Applied Physics, 47(41), [415005]. https://doi.org/10.1088/0022-
\end{abstract} $3727 / 47 / 41 / 415005$

Published in:

Journal of Physics D: Applied Physics

Document Version:

Peer reviewed version

Queen's University Belfast - Research Portal:

Link to publication record in Queen's University Belfast Research Portal

Publisher rights

This is the accepted manuscript version. The final version can be found here: http://iopscience.iop.org/0022-3727/47/41/415005/

\section{General rights}

Copyright for the publications made accessible via the Queen's University Belfast Research Portal is retained by the author(s) and / or other copyright owners and it is a condition of accessing these publications that users recognise and abide by the legal requirements associated with these rights.

Take down policy

The Research Portal is Queen's institutional repository that provides access to Queen's research output. Every effort has been made to ensure that content in the Research Portal does not infringe any person's rights, or applicable UK laws. If you discover content in the Research Portal that you believe breaches copyright or violates any law, please contact openaccess@qub.ac.uk. 


\title{
Influence of strain and polycrystalline ordering on magnetic properties of high moment rare earth metals and alloys
}

\author{
G. Scheunert ${ }^{1,3 *}$, C. Ward ${ }^{1}$, W. R. Hendren ${ }^{1}$, A. A. Lapicki², R. Hardeman², M. Mooney ${ }^{2}$, M. Gubbins ${ }^{2}$, \\ and R. M. Bowman ${ }^{1}$
}

${ }^{1}$ Centre for Nanostructured Media, School of Mathematics and Physics, Queen's University of Belfast, Belfast BT7 1NN, UK

${ }^{2}$ Seagate Technology (Ireland), Springtown Industrial Estate, 1 Disc Drive, Derry BT48 0BF, UK

${ }^{3}$ Dept. of Physics of Complex Systems, Weizmann Institute of Science, 76100 Rehovot, ISRAEL

\begin{abstract}
Despite being the most suitable candidates for solenoid pole pieces in state-of-the-art superconductorbased electromagnets, the intrinsic magnetic properties of heavy rare earth metals and their alloys have gained comparatively little attention. With the potential of integration in micro- and nanoscale devices, thin films of Gd, Dy, Tb, DyGd and DyTb were plasma-sputtered and investigated for their in-plane magnetic properties, with an emphasis on magnetisation vs. temperature profiles. Based on crystal structure analysis of the polycrystalline rare earth films, which consist of a low magnetic moment FCC layer at the seed interface topped with a higher moment HCP layer, an experimental protocol is introduced which allows the direct magnetic analysis of the individual layers. In line with the general trend of heavy lanthanides, the saturation magnetisation was found to drop with increasing unit cell size. In-situ annealed rare earth films exceeded the saturation magnetisation of a high-moment $\mathrm{Fe}_{65} \mathrm{Co}_{35}$ reference film in the cryogenic temperature regime, proving their potential for pole piece applications; however as-deposited rare earth films were found completely unsuitable. In agreement with theoretical predictions, sufficiently strained crystal phases of $\mathrm{Tb}$ and $\mathrm{Dy}$ did not exhibit an incommensurate magnetic order, unlike their single-crystal counterparts which have a helical phase. DyGd and DyTb alloys followed the trends of the elemental rare earth metals in terms of crystal structure and magnetic properties. Inter-rare-earth alloys hence present a desirable blend of saturation magnetisation and operating temperature.
\end{abstract}

Corresponding author:* gunther.scheunert@weizmann.ac.il 


\section{Introduction}

A classical electromagnet consists of $\mathrm{Cu}$ wires wound around a soft iron core with an air gap, in which the actual magnetic field $H_{a}$ is generated. The poles of the soft core at the air gap are tapered and made from material with large magnetisation $M$, which allows a large flux density $B$, and hence maximal output field strength: $H_{a} \sim B=\mu_{0}(H+M)$ [1]. Many applications requiring large magnetic fields $\left(\mu_{0} H_{a}>3 \mathrm{~T}\right)$, such as magnetic resonance imaging, electron microscope lenses or particle accelerators, use field generators based on solenoids made from type-II NbTi superconductors [2][3]. For most applications which require moderate fields of $\mu_{0} H_{a} \approx 2 \mathrm{~T}$, iron-cobalt alloys are the material of choice for pole pieces, particularly in the area of micro- and nanoscale electromagnets used in magnetic recording [4]. The $\mathrm{Fe}_{65-70} \mathrm{Co}_{35-30}$ composition in particular combines an exceptionally large saturation magnetisation with softness over a vast temperature range [5], i.e. only small $H$ fields in the milli-Tesla range of the $\mathrm{Cu}$-based solenoid are needed to achieve large magnetisation response $M$ of the pole piece [6]. However, for superconducting solenoids the core and pole material do not need to be particularly soft, since the solenoid's $H$ field is in the range of several Tesla. Thus, heavy rare earth (RE) metals can serve as alternative pole piece materials, owing their much larger saturation magnetisation [7] [8], as illustrated in figure 1.

For ultra-low temperature applications $(T \rightarrow 0 \mathrm{~K})$ holmium is most popular [9], however its magnetisation quickly drops with increasing temperature. For higher temperatures in the cryogenic regime other RE metals, particularly $\mathrm{Tb}$ and Dy, have a much more desirable magnetisation profile. Most of the available studies are conducted on single-crystal specimen, which are unsuitable for large-scale industrial fabrication, and polycrystalline material is much closer to real-device application [10]. Compared to their single-crystal counterparts, polycrystalline bulk specimen of RE metals were found to suffer from reduced saturation magnetisation [11] [12] - so do polycrystalline thin films [13] [14]. Similarly well-known but

barely investigated for thin films is the dilution of magnetic moments in inter-RE alloys and the effects on the Curie temperature [15] [16] [17]. Those alloys present a possible way to tailor magnetic properties depending on the operating temperature of the superconducting solenoid [18].

This study presents a systematic investigation on polycrystalline thin films of high magnetic moment RE metals in an attempt to understand different factors that contribute to the reduction of the saturation magnetisation in those materials and how to diminish them. Thin films are a desirable material system because of their simplicity when analysing the saturation magnetisation, with the demagnetisation factor being zero for in-plane magnetometry measurements, the capability of co-sputtering to produce high quality inter-RE alloys and efficiently protecting the films from corrosion with appropriate capping 
layers. A fabrication and analysis protocol is introduced which allows the estimation of the individual magnetisation of different crystal phases in the thin films. All RE metals shared similar crystal growth modes and trends in their magnetic properties - most importantly a reduction of the high-field magnetisation $M_{S}$ compared to single-crystal saturation magnetisation due to strain and the polycrystalline structure of the thin films.

\section{Fabrication}

Trilayer stacks of Ta $(5 \mathrm{~nm}) / \mathrm{RE} / \mathrm{Ta}(5 \mathrm{~nm})(\mathrm{RE}=\mathrm{Tb}$, DyGd, DyTb) were DC magnetron co-sputtered on $\mathrm{Si}$ and $\mathrm{Si} / 300 \mathrm{~nm}$ thermal $\mathrm{SiO}_{2}$ wafers at room temperature and on substrates heated to $350^{\circ} \mathrm{C}$, i.e. in-situ annealed. The thickness of the RE layer was $5 \mathrm{~nm}$, 50nm and $100 \mathrm{~nm}$; measured via X-ray reflectivity (XRR) as well as transmission electron microscopy (TEM) on lamellas, i.e. depth profiles which were cut from the final Ta sandwiched films via focused-ion beam (FIB). For better comparison similar deposition rates were used for all materials, which correspond to an applied power of $\sim 100 \mathrm{~W}$ per each circular threeinch target. Base pressure was maintained at $\sim 10^{-8}$ mbar for heated depositions and $\sim 10^{-9} \mathrm{mbar}$ for depositions at room temperature. The argon process gas pressure was kept constant at $4 \times 10^{-3} \mathrm{mbar}$ during deposition - high enough to allow proper plasma formation but as low as possible to diminish argon implantation in the final film. Film quality was checked post deposition for additional contaminants such as $\mathrm{O}_{2}$ and $\mathrm{N}_{2}$ with Auger-electron spectroscopy (AES) and energy-dispersive X-ray spectroscopy (EDX) with a resolution limit of $\sim 2 \%$. For co-sputtering the involved targets were placed at equal distance $(16 \mathrm{~cm})$ and orientation towards the wafer $\left(15^{\circ}\right.$ between the surface of the target and that of the wafer), itself rotating at 20RPM, to improve layer uniformity. Alloys were sputtered at elevated temperatures to assure equilibrium phase formation.

Four different $\mathrm{SiO}_{2}: \mathrm{Ta}(5 \mathrm{~nm}) / \mathrm{Tb} / \mathrm{Ta}(5 \mathrm{~nm})$ trilayer stack types were sputtered: Type $\left(\mathrm{A}^{*}\right)$ and $(\mathrm{A})$ at room temperature with $\mathrm{Tb}$ thickness of $50 \mathrm{~nm}$ and $100 \mathrm{~nm}$, respectively; and Type $\left(\mathrm{B}^{*}\right)$ and $(\mathrm{B})$ at elevated substrate temperatures of $350^{\circ} \mathrm{C}$ and again with $\mathrm{Tb}$ thickness of $50 \mathrm{~nm}$ and $100 \mathrm{~nm}$, respectively. A reference layer of $\mathrm{SiO}_{2}: \mathrm{Ta}(5 \mathrm{~nm}) / \mathrm{Tb}(5 \mathrm{~nm}) / \mathrm{Ta}(5 \mathrm{~nm})$ was sputtered at room temperature and is labelled Type (R).

Inter-RE alloys were sputtered as stacks of $\mathrm{SiO}_{2}: \mathrm{Ta}(5 \mathrm{~nm}) / \mathrm{Dy}_{50} \mathrm{Gd}_{50} / \mathrm{Ta}(5 \mathrm{~nm})$ and $\mathrm{Si}: \mathrm{Ta}(5 \mathrm{~nm}) / \mathrm{Dy}_{50} \mathrm{~Tb}_{50} / \mathrm{Ta}(5 \mathrm{~nm})$. Note, different substrates for DyGd and DyTb were chosen for this 
publication to illustrate a deviation of the initial virgin magnetisation branch in the hysteresis loop recorded at $T=4 \mathrm{~K}$ when using Si substrates.

An $\mathrm{Fe}_{65} \mathrm{Co}_{35}$ layer was sputtered at room temperature to serve as a reference, for it represents the current material of choice for room temperature high-magnetisation applications such as in magnetic recording devices [19]. The stack composition was: $\mathrm{SiO}_{2}: \mathrm{NiFe}(1 \mathrm{~nm}) / \mathrm{Fe}_{65} \mathrm{Co}_{35}(101.5 \mathrm{~nm})$.

\section{Experimental results and discussion}

X-ray diffraction (XRD) crystal structure analysis was done at room temperature; both $\Theta-2 \Theta$ and grazing incidence scans; the latter at an incoming angle of $3^{\circ}$. Three different crystal structures were identified: face-centred cubic (FCC) and two hexagonal phases. Those are labelled HCP, which was single-crystal like hexagonal-close packed, and HCP*, which corresponds to a strained hexagonal phase with an increased unit cell size.

In-plane magnetometry was conducted with a commercial Quantum Design MPMS ${ }^{\circledR}$ superconducting quantum interference device (SQUID) which could generate a maximum field strength of $\mu_{0} H=5 \mathrm{~T}$. Magnetisation measurements in this maximum field are referred to as "high-field magnetisation" or "maximal magnetisation" throughout this study. Samples for magnetometry were cut in rectangular shape with dimensions of ca. $5 \times 5 \mathrm{~mm}^{2}$ and the exact surface area was determined by optical imaging. The relative uncertainty of the magnetisation measurements is $6 \%$, whereas the relative error of the surface area measurement contributed $1 \%$, the thickness measurement contributed $3 \%$, and the magnetic moment measurement contributed $2 \%$ owing the comparatively thick $\mathrm{Si}$ and $\mathrm{SiO}_{2}$ substrates. The magnetic response of the substrates was measured separately and subtracted from the measurements of the stacks.

\section{Chemical, structural and magnetic similarity of $\mathrm{Gd}, \mathrm{Tb}$ and $\mathrm{Dy}$}

Results on the $\mathrm{SiO}_{2}: \mathrm{Ta}(5 \mathrm{~nm}) / \mathrm{Tb} / \mathrm{Ta}(5 \mathrm{~nm})$ system, as presented in this study, shall be used to elucidate the general trend of heavy RE metal thin films in terms of crystal growth modes and dependencies of the magnetic properties. Results on similar trilayer stacks of Gd have been the topic of [13] and Dy was discussed in [14]. Relevant experimental results (crystal structure and in-plane magnetometry) of comparable samples of Gd and Dy in Ta trilayer stacks are also given in this work for quicker 
comparison. Those samples which are listed were sputtered under the same conditions as $\mathrm{Tb}$ for this study with similar deposition rate.

The chemical similarity of heavy RE metals Gd, Tb and Dy, being neighbours in the lanthanide series, leads to identical crystal structure, i.e. hexagonal (nearly) close-packed (HCP) for the single crystal specimen at room temperature with very similar lattice parameters [20]. All three metals have a large magnetic moment per atom, owing their unpaired $4 f$ electrons, and a ferromagnetic phase which occurs well below room temperature, except for Gd [21].

Zero-field-cooled - field-cooled (ZFC-FC) magnetisation vs. temperature plots of all three heavy RE metals in small applied fields $\left(\mu_{0} H \rightarrow 0 \mathrm{~T}\right)$ are a common tool to understand their magnetic structure, for instance to find the easy axis and to identify the transition temperatures. Single crystal Gd is paramagnetic above and considered "simply" ferromagnetic below its Curie point of $T_{C}{ }^{G d}=293 \mathrm{~K}$. However it undergoes a spin-reorientation, i.e. the easy axis rotates with temperature and is not parallel to the $c$-axis of the hexagonal crystal any longer for $80 \mathrm{~K}<T<240 \mathrm{~K}$. It is worth noting, that there is a growing number of studies suggesting a more complex order reminiscent of the commensurate one of Er for the temperature range between the upper spin reorientation transition and $T_{C}{ }^{G d}[22]$ [23]. Tb and Dy have three distinct temperature regimes in their magnetic structure: they are ferromagnetic below their first magnetic transition temperature $\left(T_{M}{ }^{D y}=85 \mathrm{~K}, T_{M}{ }^{T b}=221 \mathrm{~K}\right)$ with the easy direction being in the basal plane, then show incommensurate helical ordering resulting in antiferromagnetism up to the second magnetic transition temperature $\left(T_{N}{ }^{D y}=179 \mathrm{~K}, T_{N}{ }^{T b}=229 \mathrm{~K}\right)$, and eventually turn paramagnetic for higher temperatures [7] [24].

\section{Crystal structure of the Ta seed layer}

Fully crystallised Ta orders body-centred cubic (BCC) with $a=3.30 \AA$ [25]. Ta seed layers in this study, of thickness $t^{T a}=5 \mathrm{~nm}$ deposited directly on $\mathrm{Si}$ or $\mathrm{SiO}_{2}$ substrates, were found to be predominantly amorphous. There might be traces of the body-centred tetragonal (BCT) phase, known for thicker layers $\left(t^{T a}>20 \mathrm{~nm}\right)$ [26] [27], but cannot be unambiguously quantified due to an overlap of its $\left(\begin{array}{lll}0 & 0 & 2\end{array}\right)$ peak with a substrate-related peak at $2 \Theta \approx 34^{\circ}$.

\section{Crystal structure of heavy rare earth metal thin films: $G d, D y, T b$}


The crystallisation behaviour of $\mathrm{Ta}(5 \mathrm{~nm}) / \mathrm{RE} / \mathrm{Ta}(5 \mathrm{~nm})$ trilayer stacks $(\mathrm{RE}=\mathrm{Gd}, \mathrm{Tb}, \mathrm{Dy})$ sputtered at room temperature and on heated substrates at moderate deposition rates $(\xi=1-3 \AA / s)$ is depicted in figure 2. In accordance with initial reports on thin-film growth dynamics of C-sandwiched RE metals by Curzon and Chlebek [28], the Ta-sandwiched RE metal thin films started with an FCC phase with a $\left(\begin{array}{lll}1 & 1 & 1\end{array}\right)$ texture at the seed interface, i.e. the (llll 111$)$ plane was parallel to the wafer surface. A likely origin are stacking faults due to lattice mismatch [29]. The RE film then turned into a HCP phase with further crystallisation. What is known for Dy, that it has an expanded HCP lattice if deposited on a seed material with a smaller lattice parameter [14], applies to all heavy RE metals under investigation as elucidated in table 1. Therefore, the FCC phase most probably forms at the Ta seed interface as it allows the largest interatomic spacing, having minimal differences in the stacking order compared to HCP [30]. If there is no stress relief during the thin-film growth, as for room temperature depositions, the film turns HCP*, depicted in figure 2 (a), which corresponds to a slightly strained hexagonal phase (compared to single crystals), however with smaller lattice parameters than the FCC phase. For the HCP* phase the $c / a$ ratio was similar to the HCP phase, i.e. the unit cell was isotropically strained, regardless of the crystallite orientation within the RE film. If additional energy in form of heating the substrate allows better crystallisation, the RE thin film changes from the FCC phase directly into the unstrained single-crystal HCP phase with a strong $\left(\begin{array}{lll}0 & 0 & 2\end{array}\right)$ texture, illustrated in figure $2(b)$.

The crystallisation behaviour was primarily deduced from XRD $\Theta-2 \Theta$ plots such as in figure 3 (a). A dominating HCP ( $\left.\begin{array}{lll}0 & 0 & 2\end{array}\right)$ peak for thicker RE films of heated depositions is accompanied by a comparatively small HCP (1 $\left.\begin{array}{lll}1 & 0\end{array}\right)$ peak and one which corresponds to FCC (lll 111$)$. Room temperature depositions have HCP peaks shifted to smaller angles, which corresponds to a strained HCP* phase. Type (R) layers only show an FCC RE phase, clamped by the Ta cap and seed.

\begin{tabular}{|c|c|c|c|c|c|}
\hline structure $\rightarrow$ & \multirow{2}{*}{\multicolumn{2}{|c|}{ CUBIC }} & \multirow{2}{*}{\multicolumn{2}{|c|}{ HCP* }} & \multirow{3}{*}{\begin{tabular}{|c|}
$\mathbf{H C P}$ \\
$a_{H C P}=3.60 \AA$ \\
\end{tabular}} \\
\hline$\downarrow$ erement & & & & & \\
\hline Gd & $a_{F C C}=5.33 \AA$ & $a_{F C C}{ }^{n}=3.77 \AA \quad \varepsilon=4.7 \%$ & $a_{H C P^{*}}=3.66 \AA$ & $\varepsilon=1.7 \%$ & \\
\hline Tb & $a_{F C C}=5.28 \AA$ & $a_{F C C}{ }^{n n}=3.73 \AA \quad \varepsilon=3.6 \%$ & $a_{H C P *}=3.64 \AA$ & $\varepsilon=1.1 \%$ & $a_{H C P}=3.60 \AA$ \\
\hline Dy & $a_{F C C}=5.21 \AA$ & $a_{F C C}{ }^{n n}=3.68 \AA \quad \varepsilon=3.1 \%$ & $a_{H C P *}=3.64 \AA$ & $\varepsilon=2.0 \%$ & $a_{H C P}=3.57 \AA$ \\
\hline$T a$ & $a_{B C C}=3.30 \AA$ & \multicolumn{4}{|c|}{$\rightarrow \rightarrow \rightarrow$ decreasing interatomic spacing $\rightarrow \rightarrow \rightarrow$} \\
\hline
\end{tabular}

Table 1: Elucidation of the decrease in interatomic spacing for the different crystal structures FCC, $H_{C P}^{*}$ and HCP in the heavy RE metal thin film investigated in this study. HCP and HCP* correspond to the single-crystal like HCP phase and one which is slightly strained, respectively. Note, $a_{F C C}{ }^{n n}=a_{F C C} / \sqrt{ } 2$ is the FCC nearest-neighbour distance and corresponds to $a_{H C P}$. Strain of the HCP* and FCC phases is given as relative change of the lattice parameter compared to the single-crystal equilibrium HCP phase $\left(\varepsilon=\Delta a / a_{H C P}\right)$. 
Although ambient temperature depositions of layers of 50nm thickness all share the same trend of the $\mathrm{HCP}^{*}$ phase changing its texture from $\left(\begin{array}{lll}0 & 0 & 2\end{array}\right)$ to $\left(\begin{array}{lll}1 & 0 & 1\end{array}\right)$ with increasing thickness (see grazing incidence surface scans in figure 3 (b), Type (A*) branch), it has to be noted that this effect seems not to prevail for thicker layers for all RE metals - Tb returned to a $\left(\begin{array}{lll}0 & 0 & 2\end{array}\right)$ texture as can be seen from XRD pattern (see grazing incidence scans in figure 3 (b), Type (A) branch). Both hexagonal phases, HCP* and HCP, were nanocrystalline for all RE metals with crystallite sizes of $D^{H C P^{*}} \approx 20 \mathrm{~nm}$ and $D^{H C P} \approx 30 \mathrm{~nm}$, respectively.

Another common feature is the limited thickness of the FCC layer. The FCC peak intensity in figure 3 (a) decreases with increasing RE film thickness, i.e. at a certain threshold thickness the RE film stops ordering FCC, turns HCP* or HCP and keeps growing only in that phase. The FCC threshold is larger for room temperature depositions and can be significantly reduced by heating the substrate during fabrication. The FCC phase of Gd thin films was investigated more closely [31] and it was found that the threshold thickness can be reduced by post-annealing (in vacuum) [32] as well as heating the substrate during deposition (i.e. in-situ annealing) [33]. The XRD spectra of Tb thin films in figure 3 suggest an FCC layer thickness of about $5 \mathrm{~nm}$ for the heated deposition and $10 \mathrm{~nm}$ for the room temperature one - similar to previous findings on Gd [33] and Dy [14].

Both hexagonal phases, HCP* and HCP, were nanocrystalline for all RE metals, whereas TEM imaging of the FCC layer did not show crystallite boundaries, hence it is likely a continuous film of limited thickness (see TEM imaging of Ta/Gd/Ta trilayers in [13] or the discussion on $\mathrm{Ta} / \mathrm{Dy}(5 \mathrm{~nm}) / \mathrm{Ta}$ stacks in [14]). This growth mode is reminiscent of Stranski-Krastanov type behaviour with the FCC phase resembling the wetting layer [34]. Results for the lattice parameters of Gd and Dy in Ta trilayer stacks are given in table 2 (a) and (b), respectively. Those for a Type (B*) and (B) Tb film, deduced from the XRD plots in figure 3, are listed in table 2 (c).

\section{Protocol for determining the saturation magnetisation of the $\mathrm{HCP}$ and $\mathrm{HCP} *$ phase}

Largest high-field magnetisation, close to single-crystal saturation, was found only for heated depositions of the RE metal thin films including Tb. For Gd it was initially only speculated [13] and later predicted [33] that this might be to do with the formation of the FCC phase at the seed interface. For Dy the otherwise metastable FCC phase was successfully clamped in a $5 \mathrm{~nm}$ film, in a trilayer stack such as Type (R), and the measured saturation magnetisation was well below saturation of single crystals [14]. The high-field magnetisation of Dy thin films with thickness beyond the FCC threshold was larger for thicker 
layers, which confirmed that the seed layer FCC phase was constant in thickness and a major source for reduced magnetisation of the overall film.

With our present understanding of the $\mathrm{Ta} / \mathrm{RE} / \mathrm{Ta}$ trilayer stack structure as presented in figure 2 , it is possible to deduce the high-field magnetisation of the $\mathrm{HCP} *$ and $\mathrm{HCP}$ phase by comparing two sets of trilayer stacks with RE layer thickness well above the FCC threshold, which was about 5nm for heated depositions and $10 \mathrm{~nm}$ for room temperature ones, therefore layers of $50 \mathrm{~nm}$ and $100 \mathrm{~nm}$ of $\mathrm{Tb}$ were sputtered. To estimate the magnetisations, the stack design was simplified to perfectly symmetric films sharing perfect interfaces as illustrated in figure 4 , which allows the use of layer thickness $\left(t_{1}, t_{2}\right)$ rather than volume $\left(V_{l}, V_{2}\right)$ for the calculations.

The magnetisation of any material is defined as magnetic moment per unit volume, which yields for our trilayer stacks with the above simplifications

$$
M_{\mathrm{all}}=\frac{M_{1} t_{1}+M_{2} t_{2}}{t_{1}+t_{2}}=\frac{M_{1} t_{1}+M_{2}\left(t-t_{1}\right)}{t_{1}+t-t_{1}}=M_{2}+\frac{t_{1}}{t}\left(M_{1}-M_{2}\right)
$$

where $M_{\text {all }}$ is the magnetisation and $t$ is the thickness of the entire RE film; $M_{l}, M_{2}$ and $t_{l}, \mathrm{t}_{2}$ are the corresponding counterparts of the individual phases as depicted in figure 4.

Solving the above equation for $M_{2}$ thus allows to calculate the HCP* or HCP layer magnetisation directly, when comparing an according set of similarly fabricated samples, only differing in their overall thickness $t$. This is possible since the magnetisation $M_{l}$ and thickness $t_{l}$ of the FCC phase is constant as long as the overall layer thickness is well above the critical threshold. It should be noted that the actual measurement of $M_{l}$ is challenging, due to possible magnetically dead layers at the Ta interfaces (due to hybridisation or pinning of interface-adjacent layers, such as reported before for Gd/Mo multilayers [35]) as well as a presumably more gradual transition to adjacent phases, stretching over a couple of nanometres [30].

For high-field magnetisation values $\mu_{0} M_{S}$ of Tb thin films as listed in table 2 (c) one gets for heated depositions: $\mu_{0} M_{2}{ }^{T b}=3.29 \mathrm{~T}$, which corresponds to $M^{H C P} / M_{0}=97 \%$ of the single-crystal saturation magnetisation $M_{0}$, and for room temperature depositions: $\mu_{0} M_{2}{ }^{T b}=2.45 \mathrm{~T}$, which is $M^{H C P^{*}} / M_{0}=72 \%$ of the single-crystal value. Applying the above protocol to Dy (high-field magnetisation values see [14]) yields a maximal magnetisation of $M^{H C P} / M_{0}=93 \%$ for the HCP phase. Gd is unique since its overall high-field magnetisation $M_{\text {all }}{ }^{G d}$ is already remarkably close to saturation $M_{\text {all }}{ }^{G d} / M_{0}=98 \%$ (see [13]) and direct measurements of pure $\mathrm{HCP}$-phase specimen in $\mathrm{Y} / \mathrm{Gd} / \mathrm{Y}$ trilayer stacks by Kwo et al. confirmed saturation magnetisation values, i.e. $M^{H C P} / M_{0} \approx 1$ [36]. 


\section{High-field magnetisation of heavy rare earth metal thin films: Gd, Dy, Tb}

From the above estimations of the high-field magnetisations of the individual layers in the RE thin films one can directly conclude that the unstrained quasi-single-crystal HCP phase is the most desirable one in terms of high-magnetisation applications. When compared with single-crystal saturation, relative magnetisations drop with increasing crystal anisotropy of the materials: the HCP phase in Gd has essentially saturation magnetisation, Tb is slightly reduced, and Dy has the lowest $M^{H C P} / M_{0}$ ratio. The maximal magnetisations of the HCP phase are the same as for bulk polycrystalline specimen which underwent annealing, e.g. as reported before by Stepankin et al. for textured Dy [12]. All values are slightly higher than early predictions of Miwa and Yosida [37] on the reduction of magnetisation in large applied fields due to polycrystalline structure and randomly aligned easy axes. All thin films had a strong texture, with the $c$-axis of the hexagonal crystal lattice along the film normal. This corresponds to the easy plane of $\mathrm{Tb}$ and Dy single crystals (for $T<T_{M}$ ), therefore these RE thin films had slightly larger high-field (in-plane) magnetisations than their polycrystalline bulk counterparts with random texture. Although the $c$-axis is the easy axis for $\mathrm{Gd}$ (for $T \rightarrow 0 \mathrm{~K}$ ), its overall crystal anisotropy is very low, which makes it much less susceptible to saturation issues due to crystallographic texture. A summary of the high-field magnetisation vs. temperature profiles of all RE metals under investigation (including DyGd and DyTb), which were sputtered on heated substrates with a RE thickness of $50 \mathrm{~nm}$, is shown in figure 5 (a). The depicted graph is a plot of $M_{\text {all }}$ and relative losses compared to single-crystal saturation. Threshold temperatures at which the $\mathrm{RE}$ magnetisations match the $\mathrm{Fe}_{65} \mathrm{Co}_{35}$ reference are indicated.

Each of the introduced heavy RE metals is a suitable candidate for a pole piece of a superconducting solenoid, including the alloys. Tb is the material of choice when aiming for reasonably high magnetisation at moderate temperatures [38] and Dy has the largest high-field magnetisation at $T \rightarrow 0 \mathrm{~K}$. It is worth noting that only Dy films had larger magnetisation than $\mathrm{Fe}_{65} \mathrm{Co}_{35}$ in moderate field strengths of $\mu_{0} H_{a} \approx 2 \mathrm{~T}$ (at $T=4 \mathrm{~K}$ ) [14], which could be generated by non-superconducting $\mathrm{Cu}$ coils with a soft Fe core.

The strained $\mathrm{HCP}^{*}$ phase has significantly lower maximal magnetisation for all RE metals. This is also reflected in the high-field magnetisation vs. temperature profile of room temperature depositions of Gd, $\mathrm{Tb}$ and Dy in figure 5 (b). None of these films are suitable alternatives to the $\mathrm{Fe}_{65} \mathrm{Co}_{35}$ reference layer. However, a major contribution for the reduction of the overall magnetisation $M_{\text {all }}$ in those films is the increased FCC layer thickness, roughly twice as much as for heated depositions. But taking the example of $\mathrm{Tb}$, where the relative loss of the $\mathrm{HCP}^{*}$ phase was $28 \%$, and bearing in mind the even larger crystal 
anisotropy of Dy, it can be concluded that none of the specimen deposited at room temperature is a suitable pole-piece candidate, even if the FCC phase is completely diminished. In how far the strained hexagonal lattice causes the moment drop is hard to quantify, but it is reasonable to assume strain to be a major contributor. Comparing the qualitative shape of the high-field magnetisation profiles for $\mathrm{Gd}$ depositions at ambient and elevated temperature reveals a distinct change in the magnetic structure, which does not occur in that form for $\mathrm{Dy}$ and $\mathrm{Tb}$. Inter- and additional intragrain anisotropies were found to impact significantly on the magnetisation behaviour (particularly in small applied fields) of comparable nanocrystalline bulk Gd [39] [40] [41] [42].

The FCC phase was shown to have even lower high-field magnetisation, e.g. for Gd [33] and for Dy [14], each time with $\mu_{0} M_{S}^{F C C}<2 \mathrm{~T}$. A similarly low value can be calculated for Tb, assuming an FCC layer thickness of $5 \mathrm{~nm}$ for the heated deposition and 10nm for the room temperature one, as suggested by the XRD spectra in figure 3. Since the FCC phase is not nanocrystalline, the differences in the atomic ordering, i.e. the inter-atomic spacing, must be the main cause of the magnetic moment reduction.

Hence the following general trend can be concluded: the high-field magnetisation of RE metals drops with increasing strain in the HCP phase, i.e. an expansion of the hexagonal lattice. The FCC phase in this context can be understood as the phase with largest lattice expansion as elucidated in table 1 . This result follows the general trend of magnetic moments dropping with increased unit cell size $V^{H C P}$ for the heavy RE metals of the lanthanide series with decreasing atomic number (caused by the "lanthanide contraction" [43]), i.e. $m_{G d}<m_{T b}<m_{D y}$ are concomitant with $V_{G d}{ }^{H C P}>V_{T b}{ }^{H C P}>V_{D y}{ }^{H C P}$ [21]. It was shown that the opposite - pressure-induced lattice contraction - causes indeed a small increase in the spontaneous magnetic moment in the ferromagnetic phase of Gd, Tb and Dy [44]. However, this moment improvement has limits and for too strong pressure, i.e. lattice contraction, the ferromagnetic structure of polycrystalline heavy RE metals breaks down and the moment rapidly decreases [45].

\section{Further magnetic properties of pure heavy rare earth metal thin films}

Figure 6 (a) shows hysteresis loops of $\mathrm{Tb}$ films Type (A) and (B) recorded at $T=4 \mathrm{~K}$. The coercive field strength is far larger for room temperature deposits: $H_{C}{ }^{(A)} / H_{C}{ }^{(B)}=3.6-$ see table 2 (c). Similar behaviour was observed for Dy $\left(H_{C}{ }^{(A)} / H_{C}{ }^{(B)}=5.2\right)$ and $\mathrm{Gd}\left(H_{C}{ }^{(A)} / H_{C}{ }^{(B)}=3.1\right)$. There is a noticeable increase in the $H_{C}{ }^{(A)} / H_{C}{ }^{(B)}$ ratio with increasing crystal anisotropy, being particularly strong for Dy.

Magnetic transition temperatures for heated depositions were found to be close-match with single-crystal transitions (easy axis) for Gd and Dy. Tb followed that trend as can be seen from ZFC-FC plots in figure 
6 (b) red branch. The transition temperatures extracted from $1 / \chi$ plots listed in table 2 (c), are also slightly below their single-crystal counterparts. Possible origin for slightly altered transition temperatures could be the nanocrystalline structure of the HCP phase [46][47], minimal contamination of the samples or simply the measurement technique in use [48]. The in-plane magnetic structure in general is reminiscent of the single-crystal easy axis, however with less distinct transitions in the ZFC-FC plots, likely a result of the polycrystalline ordering: despite being textured in a favourable way (basal plane of the hexagonal crystal $=$ in the plane of the thin film), there are still enough misaligned crystallite easy axes.

Room temperature depositions of Gd showed significant reduction of the Curie point with increased strain [33]. Dy however exhibited unusual magnetic structure for room temperature depositions, with the antiferromagnetic (helical) phase being absent, resulting in a direct transition from para- to ferromagnetism at $T_{C}=172 \mathrm{~K}$ [14]. A similar behaviour is observed for $\mathrm{Tb}$, were the antiferromagnetic phase was effectively suppressed, resulting in a direct transition to ferromagnetism at $T_{C}=216 \mathrm{~K}$, see figure 6 (b) black branch.

It can be summarised that the polycrystalline structure affects the magnetic structure by smoothing the magnetic transitions and is the most likely origin for lower transition temperatures in the unstrained HCP phase compared to single-crystal specimen. Strain causes severe changes in the magnetic structure, such as for room temperature depositions of $\mathrm{Tb}$ and $\mathrm{Dy}$, where the helical phase is completely suppressed. For Gd with a comparatively simple magnetic structure and low crystal anisotropy, polycrystalline ordering had little influence on the saturation behaviour and, for films deposited at room temperature, mainly the expanded lattice lowered the Curie temperature. It should be noted that also the opposite has been reported before, where strain effectively increased the Curie point of Gd [49]. For Dy it was shown that the FCC phase corresponds to an increasingly strained HCP* phase, with comparable magnetic structure but with lower $T_{C}$ [14]. The breakdown of the helical spin structure in Tb and Dy for the strained HCP* phase is a result of the lattice expansion, that effectively compensates the "lanthanide contraction", which is the reason of incommensurate magnetic order in heavy RE metals of higher atomic number [21].

\begin{tabular}{|c|c|c|c|}
\hline \multicolumn{4}{|c|}{$\mathrm{SiO}_{2}: \mathrm{Ta}(5 \mathrm{~nm}) / \mathrm{Gd}(50 \mathrm{~nm}) / \mathrm{Ta}(5 \mathrm{~nm})$} \\
\hline \multicolumn{2}{|c|}{ ambient deposition $\left(T=25^{\circ} \mathrm{C}, \xi=1.2 \AA / \mathrm{s}\right)$} & \multicolumn{2}{|c|}{ heated deposition $\left(T=350^{\circ} \mathrm{C}, \xi=1.2 \AA / \mathrm{s}\right)$} \\
\hline $\begin{array}{l}\text { average lattice parameter: } \\
a_{H C P^{*}}=(3.66 \pm 0.02) \AA \\
c_{H C P^{*}}=(5.82 \pm 0.02) \AA \\
a_{F C C}=(5.33 \pm 0.02) \AA\end{array}$ & $\begin{array}{l}\text { surface lattice parameter: } \\
a_{H C P^{*}}=(3.66 \pm 0.02) \AA \\
c_{H C P^{*}}=(5.82 \pm 0.02) \AA \\
\text { no FCC }(111)\end{array}$ & $\begin{array}{l}\text { average lattice parameter: } \\
a_{H C P}=(3.60 \pm 0.02) \AA \\
c_{H C P}=(5.78 \pm 0.02) \AA \\
a_{F C C}=(5.33 \pm 0.02) \AA\end{array}$ & $\begin{array}{l}\text { surface lattice parameter: } \\
a_{H C P}=(3.61 \pm 0.02) \AA \\
c_{H C P}=(5.78 \pm 0.02) \AA \\
\text { no FCC }(111)\end{array}$ \\
\hline $\begin{array}{l}\text { saturation at } T=4 \mathrm{~K}: \\
\mu_{0} M_{S}=(2.25 \pm 0.14) \mathrm{T}\end{array}$ & $\begin{array}{l}\text { transition temperature: } \\
T_{C}=(278 \pm 2) \mathrm{K}\end{array}$ & $\begin{array}{l}\text { saturation at } T=4 \mathrm{~K} \text { : } \\
\mu_{0} M_{S}=(2.61 \pm 0.16) \mathrm{T}\end{array}$ & $\begin{array}{l}\text { transition temperature: } \\
T_{C}=(293 \pm 2) \mathrm{K}\end{array}$ \\
\hline coercivity at $T=4 \mathrm{~K}$ : & remnance at $T=4 \mathrm{~K}$ : & coercivity at $T=4 \mathrm{~K}:$ & remnance at $T=4 \mathrm{~K}:$ \\
\hline
\end{tabular}




\begin{tabular}{|l|l|l|l|}
\hline$\mu_{0} H_{C}=(0.050 \pm 0.003) \mathrm{T}$ & $\mu_{0} M_{\text {rem }} \sim 0.71 \mathrm{~T}$ & $\mu_{0} H_{C}=(0.016 \pm 0.001) \mathrm{T}$ & $\mu_{0} M_{\text {rem }} \sim 1.44 \mathrm{~T}$ \\
\hline
\end{tabular}

Table 2 (a): Experimental summary, room temperature and heated depositions $-G d$.

\begin{tabular}{|c|c|c|c|}
\hline \multicolumn{4}{|c|}{$\mathrm{SiO}_{2}: \mathrm{Ta}(5 \mathrm{~nm}) / \mathrm{Dy}(50 \mathrm{~nm}) / \mathrm{Ta}(5 \mathrm{~nm})$} \\
\hline \multicolumn{2}{|c|}{ ambient deposition $\left(T=25^{\circ} \mathrm{C}, \xi=1.4 \AA / \mathrm{s}\right)$} & \multicolumn{2}{|c|}{ heated deposition $\left(T=350^{\circ} \mathrm{C}, \xi=1.4 \AA \mathrm{A} / \mathrm{s}\right)$} \\
\hline $\begin{array}{l}\text { average lattice parameter: } \\
a_{H C P^{*}}=(3.64 \pm 0.02) \AA \\
c_{H C P^{*}}=(5.70 \pm 0.02) \AA \\
a_{F C C}=(5.21 \pm 0.02) \AA\end{array}$ & $\begin{array}{l}\text { surface lattice parameter: } \\
\text { no HCP }(100) \\
\text { no HCP }(002) \\
\text { no FCC }(111)\end{array}$ & $\begin{array}{l}\text { average lattice parameter: } \\
a_{H C P}=(3.57 \pm 0.02) \AA \\
c_{H C P}=(5.65 \pm 0.02) \AA \\
a_{F C C}=(5.21 \pm 0.02) \AA\end{array}$ & $\begin{array}{l}\text { surface lattice parameter: } \\
\text { no HCP }(100) \\
c_{H C P}=(5.71 \pm 0.02) \AA \\
\text { no FCC }(111)\end{array}$ \\
\hline $\begin{array}{l}\text { saturation at } T=4 \mathrm{~K}: \\
\mu_{0} M_{S}=(2.26 \pm 0.14) \mathrm{T}\end{array}$ & $\begin{array}{l}\text { transition temperature: } \\
T_{C}=(172 \pm 2) \mathrm{K}\end{array}$ & $\begin{array}{l}\text { saturation at } T=4 \mathrm{~K}: \\
\mu_{0} M_{S}=(3.28 \pm 0.20) \mathrm{T}\end{array}$ & $\begin{array}{l}\text { transition temperatures: } \\
T_{M}=(80 \pm 2) \mathrm{K}, T_{N}=(173 \pm 2) \mathrm{K}\end{array}$ \\
\hline $\begin{array}{l}\text { coercivity at } T=4 \mathrm{~K}: \\
\mu_{0} H_{C}=(1.10 \pm 0.06) T\end{array}$ & $\begin{array}{l}\text { remnance at } T=4 \mathrm{~K}: \\
\mu_{0} M_{\text {rem }} \sim 1.18 \mathrm{~T}\end{array}$ & $\begin{array}{l}\text { coercivity at } T=4 \mathrm{~K}: \\
\mu_{0} H_{C}=(0.21 \pm 0.01) \mathrm{T}\end{array}$ & $\begin{array}{l}\text { remnance at } T=4 \mathrm{~K}: \\
\mu_{0} M_{r e m} \sim 2.31 \mathrm{~T}\end{array}$ \\
\hline
\end{tabular}

Table 2 (b): Experimental summary, room temperature and heated depositions $-D y$.

\begin{tabular}{|c|c|c|c|}
\hline \multicolumn{4}{|c|}{$\mathrm{SiO}_{2}: \mathrm{Ta}(5 \mathrm{~nm}) / \mathrm{Tb}(50 \& 100 \mathrm{~nm}) / \mathrm{Ta}(5 \mathrm{~nm})$} \\
\hline \multicolumn{2}{|c|}{ Type $\left(A, A^{*}\right)$ - ambient deposition $\left(T=25^{\circ} \mathrm{C}, \xi=1.3 \AA \mathrm{A} / \mathrm{s}\right)$} & \multicolumn{2}{|c|}{ Type $\left(\mathrm{B}, \mathrm{B}^{*}\right)$ - heated deposition $\left(T=350^{\circ} \mathrm{C}, \xi=1.3 \AA \mathrm{A} / \mathrm{s}\right)$} \\
\hline $\begin{array}{l}\text { average lattice parameter: } \\
a_{H C P^{*}}=(3.64 \pm 0.02) \AA \\
C_{H C P^{*}}=(5.76 \pm 0.02) \AA \\
a_{F C C}=(5.28 \pm 0.02) \AA\end{array}$ & $\begin{array}{l}\text { surface lattice parameter: } \\
a_{H C P^{*}}=(3.67 \pm 0.02) \AA \\
c_{H C P^{*}}=(5.79 \pm 0.02) \AA \\
a_{F C C} \sim 5.28 \AA\end{array}$ & $\begin{array}{l}\text { average lattice parameter: } \\
a_{H C P}=(3.60 \pm 0.02) \AA \\
c_{H C P}=(5.69 \pm 0.02) \AA \\
a_{F C C}=(5.25 \pm 0.02) \AA\end{array}$ & $\begin{array}{l}\text { surface lattice parameter: } \\
a_{H C P}=(3.61 \pm 0.02) \AA \\
c_{H C P}=(5.70 \pm 0.02) \AA \\
\text { no FCC }(111)\end{array}$ \\
\hline $\begin{array}{ll}\text { saturation at } T=4 \mathrm{~K}: & \\
\mu_{0} M_{S}=(2.31 \pm 0.14) \mathrm{T} & (\mathrm{A}) \\
\mu_{0} M_{S}=(2.19 \pm 0.13) \mathrm{T} & \left(\mathrm{A}^{*}\right)\end{array}$ & $\begin{array}{l}\text { transition temperature: } \\
T_{C}=(216 \pm 2) \mathrm{K}\end{array}$ & $\begin{array}{ll}\text { saturation at } T=4 \mathrm{~K}: & \\
\mu_{0} M_{S}=(3.14 \pm 0.19) \mathrm{T} & (\mathrm{B}) \\
\mu_{0} M_{S}=(3.01 \pm 0.18) \mathrm{T} & \left(\mathrm{B}^{*}\right)\end{array}$ & $\begin{array}{l}\text { transition temperatures: } \\
T_{M}=(223 \pm 2) \mathrm{K}, T_{N}=(230 \pm 2) \mathrm{K}\end{array}$ \\
\hline $\begin{array}{l}\text { coercivity at } T=4 \mathrm{~K}: \\
\mu_{0} H_{C}=(0.57 \pm 0.03) \mathrm{T}\end{array}$ & $\begin{array}{l}\text { remnance at } T=4 \mathrm{~K}: \\
\mu_{0} M_{\text {rem }} \sim 0.41 \mathrm{~T} \quad \text { (A) }\end{array}$ & $\begin{array}{l}\text { coercivity at } T=4 \mathrm{~K}: \\
\mu_{0} H_{C}=(0.16 \pm 0.01) \mathrm{T}\end{array}$ & $\begin{array}{l}\text { remnance at } T=4 \mathrm{~K}: \\
\mu_{0} M_{r e m} \sim 1.67 \mathrm{~T} \\
\end{array}$ \\
\hline
\end{tabular}

Table 2 (c): Experimental summary, room temperature and heated depositions $-T b$.

\section{Crystal structure of DyGd and DyTb}

Dysprosium-gadolinium (DyGd) and dysprosium-terbium (DyTb) follow the trend of elemental heavy RE metals. Both order hexagonal, quasi-HCP, however the lattice parameters are not a simple linear blend of the elemental values, see table 3. Again an FCC phase formed at the seed interface and disappeared with further film growth, making way for a crystalline HCP phase, see XRD $\Theta-2 \Theta$ spectra in figure 7 (a) and (b) for DyGd and DyTb, respectively. Difficulties arose in stabilising the film texture which nearly vanished for further film growth as can be seen from the poor signal of grazing incidence scans in figure 7. Those indicate the RE typical $\left(\begin{array}{lll}0 & 0 & 2\end{array}\right)$ texture for the heated deposition of DyTb, but suggest a $\left(\begin{array}{lll}1 & 0 & 1\end{array}\right)$ texture for DyGd, despite being deposited at elevated substrate temperatures. The average crystallite size was $D \approx 25 \mathrm{~nm}$ for DyGd and DyTb alike and determined from the XRD $\Theta-2 \Theta$ spectra's $\left(\begin{array}{lll}0 & 0 & 2\end{array}\right)$ peak via Scherrer analysis. Results for the lattice parameters of DyGd and DyTb are listed in table 3.

\section{In-plane magnetic properties of DyGd and DyTb}


High-field magnetisations of DyGd and DyTb present the expected blend of the magnetic moments of the involved elements with strong parallel coupling below the temperature of the ferromagnetic transition. Figure 8 (a) shows the high-field magnetisation vs. temperature profile of DyGd and figure 8 (b) the one for DyTb, each with respect to the elemental RE profiles. The magnetisation profiles share a common nexus at $T_{X}{ }^{D y G d}=154 \mathrm{~K}$ and $T_{X}{ }^{D y T b}=131 \mathrm{~K}$, for DyGd and DyTb respectively, where $M_{S}\left(T_{X}\right)$ are similar for the involved elements. Below the transition to ferromagnetism of the alloys, the high-field magnetisations lie roughly between the elemental ones, which indicates simple moment dilution. Although the DyTb magnetisation curve is not exactly in the middle, it lies well within the uncertainty range. Leaving the ferromagnetic regime, the magnetisation profiles deviate from the simple-blend central curve due to different temperature dependences of the magnetic moments within these two-component systems.

Both alloys had no simple ferromagnetic to paramagnetic transition, but an antiferromagnetic phase was found, presumably helical structure. The Néel point for DyGd lay at $T_{N}=245 \mathrm{~K}$ and can be identified from a small sharp spike in the ZFC-FC plots in figure 8 (c). This result does not completely agree with findings on single-crystal bulk DyGd, where an antiferromagnetic plateau was only observed for Dy-rich alloys [17] [50]. For DyTb the Néel spike indicated a transition at $T_{N}=194 \mathrm{~K}$, see figure 8 (d). Figures 8 (c) and (d) also give information on which RE element dominated the magnetic structure for low applied field scans: the softer Gd for DyGd and, rather unintuitive, the harder Dy for DyTb. Although the ZFCFC magnetisation vs. temperature profiles of the in-plane direction were reminiscent of easy-axis behaviour in single crystals, it could be that the anisotropy of the $\mathrm{Tb}$ atoms in the alloy does not favour that direction, hence the Dy dominance. The transition to the ferromagnetic state was determined from $1 / \chi$ plots and lay between the corresponding values of $\mathrm{Gd}$, Dy and $\mathrm{Tb}$ but was above their weighted average for DyGd with $T_{M}{ }^{D y G d}=225 \mathrm{~K}$, slightly lower than what has been reported before for single-crystals [50]. The transition occurred below the weighted average for DyTb with $T_{M}{ }^{D y T b}=130 \mathrm{~K}$. Following the above considerations for elemental RE films, the nanocrystalline structure of DyGd and DyTb may be the reason for unexpected transition temperatures, and residual strain could be a possible origin of the observed differences to single-crystal magnetic structure.

The in-plane coercivities of both specimen, recorded at $T=4 \mathrm{~K}$, were similarly large (see table 3 ) exceeding even that of comparable Dy films by $\sim 25 \%$. A less prominent reason could be the slightly smaller crystallite size [47]; however the coupling of the different RE atoms is more likely the origin of large coercivity. For $T=4 \mathrm{~K}$, single crystals of $\mathrm{Gd}$ have their easy axis along the $c$-axis in contrast to $\mathrm{Tb}$ and Dy, whose easy direction is the basal plane. Hence, DyGd with perpendicular standing easy axes of Gd and Dy has larger coercivity than DyTb, despite larger anisotropy of Tb compared to Gd. 
Another feature revealed in figure 8 (e) and (f) is an initial "virgin" magnetisation branch which lies outside the normal hysteresis loop (after first saturation) for Dy (not shown) and Dy-based alloys (compare figure 8 (e), DyGd on $\mathrm{SiO}_{2}$, with 8 (f), DyTb on $\mathrm{Si}$ ). This effect was thermally reversible, i.e. after the sample had been warmed above $T_{N}$ and cooled down in ZF the virgin branch lay again outside the normal hysteresis loop. This effect always occurred for Si wafers, although no other noticeable influence on the magnetic properties for RE metal and alloy thin films deposited on Si instead of $\mathrm{SiO}_{2}$ was found. It might be a result of a small in-plane texture, favouring e.g. the $a$-axis over the $b$-axis direction, giving rise to a small anisotropy which is overcome by saturating the specimen, reminiscent of magnetic annealing. Further investigation of this effect is beyond the scope of this study, particularly as it can simply be avoided by using $\mathrm{SiO}_{2}$ wafers instead of $\mathrm{Si}$.

\begin{tabular}{|c|c|c|c|}
\hline \multicolumn{2}{|c|}{$\mathrm{SiO}_{2}: \mathrm{Ta}(5 \mathrm{~nm}) / \mathrm{DyGd}(50 \mathrm{~nm}) / \mathrm{Ta}(5 \mathrm{~nm})$} & \multicolumn{2}{|c|}{ Si:Ta(5nm)/DyTb(50nm)/Ta(5nm) } \\
\hline \multicolumn{2}{|c|}{ heated deposition $\left(T=350^{\circ} \mathrm{C}, \xi=1.4 \AA \mathrm{A} / \mathrm{s}\right.$ each $)$} & \multicolumn{2}{|c|}{ heated deposition $\left(T=350^{\circ} \mathrm{C}, \xi=1.4 \AA \mathrm{A} / \mathrm{s}\right.$ each) } \\
\hline $\begin{array}{l}\text { average lattice parameter: } \\
a_{H C P}=(3.69 \pm 0.02) \AA \\
c_{H C P}=(5.72 \pm 0.02) \AA \\
a_{F C C}=(5.31 \pm 0.02) \AA\end{array}$ & $\begin{array}{l}\text { surface lattice parameter: } \\
a_{H C P}=(3.64 \pm 0.02) \AA \\
c_{H C P}=(5.75 \pm 0.02) \AA \\
\text { no FCC }(111)\end{array}$ & $\begin{array}{l}\text { average lattice parameter: } \\
a_{H C P}=(3.65 \pm 0.02) \AA \\
c_{H C P}=(5.67 \pm 0.02) \AA \\
a_{F C C}=(5.24 \pm 0.02) \AA\end{array}$ & $\begin{array}{l}\text { surface lattice parameter: } \\
\text { no HCP }(100) \\
c_{H C P}=(5.69 \pm 0.02) \AA \\
\text { no FCC }(111)\end{array}$ \\
\hline $\begin{array}{l}\text { saturation at } T=4 \mathrm{~K}: \\
\mu_{0} M_{S}=(2.94 \pm 0.18) \mathrm{T}\end{array}$ & $\begin{array}{l}\text { transition temperatures: } \\
T_{M}=(225 \pm 2) \mathrm{K}, T_{N}=(245 \pm 2) \mathrm{K}\end{array}$ & $\begin{array}{l}\text { saturation at } T=4 \mathrm{~K}: \\
\mu_{0} M_{S}=(3.18 \pm 0.19) \mathrm{T}\end{array}$ & $\begin{array}{l}\text { transition temperatures: } \\
T_{M}=(130 \pm 2) \mathrm{K}, T_{N}=(194 \pm 2) \mathrm{K}\end{array}$ \\
\hline $\begin{array}{l}\text { coercivity at } T=4 \mathrm{~K}: \\
\mu_{0} H_{C}=(0.28 \pm 0.01) \mathrm{T}\end{array}$ & $\begin{array}{l}\text { remnance at } T=4 \mathrm{~K}: \\
\mu_{0} M_{\text {rem }} \sim 1.80 \mathrm{~T}\end{array}$ & $\begin{array}{l}\text { coercivity at } T=4 \mathrm{~K}: \\
\mu_{0} H_{C}=(0.27 \pm 0.01) \mathrm{T}\end{array}$ & $\begin{array}{l}\text { remnance at } T=4 \mathrm{~K}: \\
\mu_{0} M_{\text {rem }} \sim 2.40 \mathrm{~T}\end{array}$ \\
\hline
\end{tabular}

Table 3: Experimental summary, heated depositions - DyGd (left column) and DyTb (right column).

\section{Summary and Outlook}

Thin films of polycrystalline heavy RE metals Gd, Tb, Dy, DyGd and DyTb were found to be suitable candidates for pole-piece applications in solenoids, having larger high-field magnetisation in the cryogenic temperature regime than a high-moment $\mathrm{Fe}_{65} \mathrm{Co}_{35}$ reference layer. However, this only applies to RE films which were deposited on heated substrates and as a consequence consisted primarily of an unstrained HCP phase, reminiscent to the equilibrium phase of single crystals, and a comparatively small, about $5 \mathrm{~nm}$ thick FCC layer at the seed interface. In contrast, thin films of Gd, Tb and Dy deposited at room temperature had significantly lower high-field magnetisation than the $\mathrm{Fe}_{65} \mathrm{Co}_{35}$ reference. Those $\mathrm{RE}$ films consisted of a strained HCP* phase and a larger, about 10nm thick FCC layer at the seed. 
Therefore, it was concluded that strain-induced lattice expansion for the HCP* and FCC phase led to a strong reduction of the magnetic moments, hence also the high-field magnetisations of the investigated heavy RE metals. The FCC phase in this context can be understood as the extreme case of maximum lattice expansion - concomitant with smallest saturation magnetisation. Unlike for $3 d$ transition metals such as Fe, increasing the interatomic spacing does not necessarily increase the magnetic moment of RE atoms. They can already be considered isolated moments, owing their large atomic radii and spontaneous moments on the well-shielded $4 f$ shell, and changes in the interatomic distance result in magnetic moment changes being subject to the oscillatory nature of the Ruderman-Kittel-Kasuya-Yoshida (RKKY) coupling [51].

At the present, $\mathrm{Fe}_{65-70} \mathrm{Co}_{35-30}$ is still the unrivalled material of choice for solenoid pole-piece applications at room temperature and none of the high-magnetisation heavy RE metals as presented in this study, including Ho, can compete. This largest ever measured saturation magnetisation of a bulk specimen at room temperature is $\mu_{0} M_{S}{ }^{F e C o}=2.45 \mathrm{~T}$ [52] - commonly referred to as the "Slater-Pauling limit".

The reduction or even suppression of the FCC and HCP* content in any polycrystalline RE specimen is crucial to fabricate high-magnetisation materials. This can best be achieved for thin films by using perfect-match RE-based seed materials, as shown for Y-seeded Gd thin films, due to compatibility of the electronic structure [36][53]. Heat treatment such as post-annealing in vacuum or in-situ annealing during fabrication, as done for this study, have also proven powerful tools to reduce the content of undesired crystal phases and are directly applicable to the fabrication of bulk specimen.

Essentially all thin films of RE metals, deposited on anything which is not a perfect-match seed layer with sufficiently matched electronic structure, show clear indication of strain and, at least, a distorted hexagonal phase, if not FCC ordering. Therefore, one should be very careful not to simply assume singlecrystal magnetic moments for RE thin films and other nanostructures [29].

The expansion of the unit cell also changed the magnetic structure, clearly visible in the strained HCP* phases in $\mathrm{Tb}$ and Dy, where according to theoretical predictions the incommensurate order was suppressed and no helical phase could be observed.

Crystallographic texture primarily played a role for $\mathrm{Tb}$ and $\mathrm{Dy}$, owing their large crystal anisotropy, and inhibited full saturation of the unstrained polycrystalline yet single-crystal like HCP phase in maximum applied fields of $\mu_{0} H=5 \mathrm{~T}$.

Heated depositions of DyGd and DyTb confirmed their similarity in terms of crystal structure and magnetic properties with elemental RE metals, ordering also HCP with a small FCC phase at the seed 
interface. A desirable blend of magnetic moments and points of magnetic transition was found, which makes inter-RE alloys suitable candidates for high-moment applications with the benefit of tailoring intrinsic magnetic properties as required. Large coercivities due to competing anisotropies were identified as a main obstacle in pole-piece applications. However, our findings also raise hope to find an inter-RE alloy composition where the anisotropies of the different RE atoms compensate [54], which may yield a particularly soft alloy, with applicability even in cheap non-superconducting solenoids.

With the introduced analysis protocol, which allows a separate investigation of the magnetic phases of the dual-phase RE films in Ta/RE/Ta, the above suggested soft inter-RE alloys can be fabricated in similar trilayer stacks even without prior knowledge of a suitable perfect-match seed material for stabilisation of the desirable high-moment HCP crystal phase. After identification of the right alloy composition, tailored to meet the magnetisation requirements of a specific temperature range, a suitable perfect-match seed material can be developed preferentially starting from other RE alloys.

\section{Acknowledgements}

We thank Seagate Technology (Ireland) for their financial support to establish ANSIN (www.ansin.eu) and help with sample analysis via TEM and AES. We would also like to thank Michael Coey (Trinity College Dublin, Ireland) for several insights on rare earth magnetism and suggestions regarding future work. Special thanks go to Dan Oron (Weizmann Institute of Science) for his support which allowed us to publish our results. 


\section{References}

[1] Ishikawa Y and Chikazumi S 1962 Design of High Power Electromagnets Jpn. J. Appl. Phys. 1155

[2] Seidel P 2015 Handbook of Applied Superconductivity (Weinheim, Bergstr: Wiley-VCH)

[3] Hott R, Kleiner R, Wolf T and Zwicknagl G 2013 Review on Superconducting Materials arXiv:1306.0429 [cond-mat.supr.con]

[4] Cooper E I, Bonhote C, Heidmann J, Hsu Y, Kern P, Lam J W, Ramasubramanian M, Robertson N, Romankiw L T and Xu H 2005 Recent developments in high-moment electroplated materials for recording heads IBM J. Res. \& Dev. 49103

[5] Sourmail T 2005 Near equiatomic FeCo alloys: Constitution, mechanical and magnetic properties Progress in Materials Science $\mathbf{5 0} 816$

[6] Cullity B D and Graham C D 2009 Introduction to Magnetic Materials (Hoboken, New Jersey: John Wiley \& Sons, Inc.)

[7] Belov K P, Levitin R Z and Nikitin S A 1964 Ferromagnetism and antiferromagnetism of rare-earth metals Soviet Physics Uspekhi 7179

[8] Rhyne J J and McGuire T R 1972 Magnetism of rare-earth elements, alloys, and compounds IEEE Trans. Magn. 8105

[9] Hoard R W, Mance S C, Leber R L, Dalder E N, Chaplin M R, Blair K, Nelson D H and Van Dyke D A 1985 Field enhancement of a 12.5-T magnet using holmium poles IEEE Trans. Magn. 21448

[10] Agustsson R, Chen Y C, Grandsaert II T, Murokh A, Solovyov V and O'Shea F 2012 Praseodymium iron-boron undulator with textured dysprosium poles for compact $x$-ray fel applications Proceedings of IPAC2012, New Orleans, Lousiana, USA MOPPP086 756

[11] Safrata R S, Fisher T R and Shelley E G 1966 Magnetic hysteresis of holmium metal at 4.2K J. Appl. Phys. 374869

[12] Stepankin V 1995 Magnetically aligned polycrystalline dysprosium as ultimate saturation ferromagnet for high magnetic field polepieces Physica B: Condensed Matter 211 345-7

[13] Scheunert G, Ward C, Hendren W R and Bowman R M 2012 Magnetization of 2.6T in gadolinium thin films Appl. Phys. Lett. 101142407

[14] Scheunert G, Hendren W R, Lapicki A A, Hardeman R, Gubbins M and Bowman R M 2013 Improved magnetization in sputtered dysprosium thin films J. Phys. D: Appl. Phys. 46152001

[15] Koehler W C 1965 Magnetic properties of rare-earth metals and alloys Journal of Applied Physics 361078 
[16] Bozorth R M, Gambino R J and Clark A E 1968 Magnetization of Some Single-Crystal Alloys of Rare Earth Metals J. Appl. Phys. 39883

[17] Yang T-T and Robinson L B 1969 Magnetic resistivity and magnetic transitions in rare-earth alloys Phys. Rev. 185743

[18] Spano M L, Clark A E and Wun-Fogle M 1990 Magnetostriction of TbDy single crystals under compressive strain IEEE Trans. Magn. 261751

[19] Jung H S, Doyle W D and Matsunuma S 2003 Influence of underlayers on the soft properties of high magnetization FeCo films J. Appl. Phys. 936462

[20] Jensen J and Mackintosh A R 1991 Rare Earth Magnetism - Structures and Excitations (Oxford: Clarendon Press)

[21] Hughes I D, Daene M, Ernst A, Hergert W, Lueders M, Poulter J, Staunton J B, Svane A, Szotek Z and Temmerman W M 2007 Lathanide contraction and magnetism in the heavy rare earth elements Nature 446650

[22] Coey J M D, Skumryev V and Gallagher K 1999 Is gadolinium really ferromagnetic? Nature 40135

[23] Kaul S N 2003 Is gadolinium a helical antiferromagnet or a collinear ferromagnet? PRAMANA journal of physics 60505

[24] Hegland D E, Legvold S and Spedding F H 1963 Magnetization and electrical resistivity of terbium single crystals Phys. Rev. 131158

[25] Face D W and Prober D E 1987 Nucleation of body-centered cubic tantalum films with a thin niobium underlayer J. Vac. Sci. Technol. A 53408

[26] Schauer A and Roschy M 1972 R.F. sputtered beta-tantalum and B.C.C tantalum films Thin Solid Films 12313

[27] Molarius J M T, Suni I, Laurila T, Zeng K and Kivilahti J K 1999 R.F.-sputtered tantalum-based diffusion barriers between copper and silicon Superficies y Vacio 9206

[28] Curzon A E and Chlebek H G 1979 The observation of face centred cubic Gd, Tb, Dy, Ho, Er and Tm in the form of thin films and their oxidation J. Phys. F: Met. Phys. 31-5

[29] Hsu C-J, Prikhodko S V, Wang C-Y, Chen L-J and Carman G P 2012 Magnetic anisotropy in nanostructured gadolinium J. Appl. Phys. 111053916

[30] Olson G B and Cohen M 1976 A general mechanism of martensitic nucleation: Part I. General concepts and the FCC $\rightarrow$ HCP transformation MTA 7 1897-904

[31] Bist B M S and Srivastava O N 1973 A new f.c.c. gadolinium phase and its oxidation J. LessCommon Met. 3399 
[32] Hussain A A A and Al-Bassam T S 1979 Effects of deposition parameters on the structure of thin gadolinium films Thin Solid Films 5977

[33] Ward C, Scheunert G, Hendren W R and Bowman R M 2013 Realizing the high moment in $\mathrm{Fe} / \mathrm{Cr} / \mathrm{Gd}$ : the role of the rare earth Appl. Phys. Lett. 102092403

[34] Baskaran A and Smereka P 2012 Mechanisms of Stranski-Krastanov growth Journal of Applied Physics 111044321

[35] Harkins J V and Donovan P E 1996 The structure and magnetization of gadolinium/molybdenum multilayers J. Phys.: Condens. Matter 8685

[36] Kwo J, McWhan D B, Hong M, Gyorgy E M and DiSalvo F J 1986 Structural and magnetic properties of single crystal rare earth Gd-Y superlattices Mat. Res. Soc. Symp. Proc. 56211

[37] Miwa H and Yosida K 1961 Magnetic ordering in the rare-earth metals with more than half-filled 4f shells Prog. Theor. Phys. 26693

[38] Paul A, Mukherjee S, Kreuzpaintner W and Böni P 2014 Exchange-bias-like coupling in a ferrimagnetic Fe/Tb multilayer Physical Review B 89

[39] Mathew S P and Kaul S N 2012 Magnetization process in nanocrystalline gadolinium J. Phys.: Condens. Matter 24256008

[40] Shand P M, Bohnet J G, Shield J E, Schmitter D, Shelburne G and Leslie-Pelecky D 2008 Magnetic behavior of melt-spun gadolinium Physical Review B 77 1-11

[41] Ryan D H, Michels A, Döbrich F, Birringer R, Yamani Z and Cadogan J M 2013 Magnetic ordering in nanocrystalline gadolinium: A neutron diffraction study Phys. Rev. B 87064408

[42] Döbrich F, Kohlbrecher J, Sharp M, Eckerlebe H, Birringer R and Michels A 2012 Neutron scattering study of the magnetic microstructure of nanocrystalline gadolinium Phys. Rev. B 85094411

[43] Taylor K N R and Darby M I 1972 Physics of Rare Earth Solids (London, UK: Chapman and Hall)

[44] Bartholin H and Bloch D 1968 Magnetic ordering temperatures of the single crystals of rare earth metals at high pressures: Gadolinium-terbium-dysprosium Journal of Physics and Chemistry of Solids 29 1063-75

[45] Mito M, Matsumoto K, Komorida Y, Deguchi H, Takagi S, Tajiri T, Iwamoto T, Kawae T, Tokita M and Takeda K 2009 Volume shrinkage dependence of ferromagnetic moment in lanthanide ferromagnets gadolinium, terbium, dysprosium, and holmium J. Phys. Chem. Solids 701290

[46] Johnson D, Perera P and O'Shea M J 1996 Finite size effects in nanoscale Tb particles J. Appl. Phys. 795299

[47] O'Shea M J and Perera P 1999 Influence of nanostructure (layers and particles) on the magnetism of rare-earth materials J. Appl. Phys. 854322 
[48] Dan'kov S Y, Tishin A M, Percharsky V K and Gschneidner Jr. K A 1998 Magnetic phase transitions and the magnetothermal properties of gadolinium Phys. Rev. B 473478

[49] Skomski R, Waldfried C and Dowben P A 1998 The influence of the surface on the spontaneous magnetization of Gd thin films J. Phys.: Condens. Matter 105833

[50] Milstein F and Robinson L B 1967 Magnetic transitions in alloys of gadolinium and dysprosium Phys. Rev. 159466

[51] Freeman A J and Wu R 1991 Magnetic moment enhancement at transition metal and rare-earth metal surfaces Prog. Theor. Phys. Supplement 106397

[52] Noma K, Matsuoka M, Kanai H, Uehara Y, Nomura K and Awaji N 2006 Ultra-high magnetic moment films for write head IEEE Trans. Magn. 42140

[53] Kwo J, Gyorgy E M, McWhan D B, Hong M, DiSalvo F J, Vettier C and Bowe J E 1985 Magnetic and structural properties of single-crystal rare-earth Gd-Y superlattices Phys. Rev. Lett. 551402

[54] Amin A Y 1979 Magnetic properties of inter-rare-earth alloys at low temperatures in high magnetic fields J. Appl. Phys. 501035

[55] Clegg D W and Buckley R A 1973 The disorder - order transformation in iron-cobalt-based alloys Metal Science Journal 748

[56] Chernyshov A S, Tsokol A O, Tishin A M, Gschneidner K A and Pecharsky V K 2005 Magnetic and magnetocaloric properties and the magnetic phase diagram of single-crystal dysprosium Phys. Rev. B 71184410

[57] Rhodes B L, Legvold S and Spedding F H 1958 Magnetic properties of holmium and thulium metals Phys. Rev. 1091547 


\section{FIGURE 1:}

Extrapolated saturation-magnetisation vs. temperature plots for bulk specimen of $\mathrm{Fe}_{65} \mathrm{Co}_{35}, \mathrm{Gd}, \mathrm{Tb}, \mathrm{Dy}$ and Ho. Qualitatively, the magnetisation vs. temperature behaviour was deduced from measurements on polycrystalline thin films with (quasi) single-crystal structure and lattice parameters, as for this study, but the maximal moments at $T \rightarrow 0 \mathrm{~K}$ were taken to be saturation moments of single crystals [20]. Confirmation for the bulk easy-axis magnetisation profile for each material can be found in literature: (polycrystalline) FeCo [55], Gd [48], Dy [56], Tb [24], (polycrystalline) Ho [57].

\section{FIGURE 2:}

Depiction of the thin-film crystal structure of RE trilayer stacks of $\mathrm{Ta}(5 \mathrm{~nm}) / \mathrm{RE}(50 \mathrm{~nm}) / \mathrm{Ta}(5 \mathrm{~nm})$ on Si or $\mathrm{SiO}_{2}$ substrates, for (a) ambient temperature depositions and (b) heated depositions. All heavy RE metals under investigation (including DyGd and DyTb) exhibited the same growth mode. A quasi-amorphous Ta seed layer on the $\mathrm{Si}$ or $\mathrm{SiO}_{2}$ substrate promoted the formation of an FCC phase of the RE. Ambient depositions had a much larger FCC content than heated depositions. With further growth, the FCC phase turned into a strained $\mathrm{HCP}^{*}$ phase for ambient depositions and a single-crystal HCP phase for heated depositions, both with a strong ( $\left.\begin{array}{lll}0 & 0 & 2\end{array}\right)$ texture. For thicker RE layers (not depicted), ambient depositions developed a ( $\left.\begin{array}{lll}1 & 0 & 1\end{array}\right)$ texture. Heated depositions had significantly larger grain sizes. To visualise the texture, red lines are drawn to indicate the $c$-axis direction for each grain.

\section{FIGURE 3:}

XRD scans of $\mathrm{SiO}_{2}: \mathrm{Ta}(5 \mathrm{~nm}) / \mathrm{Tb} / \mathrm{Ta}(5 \mathrm{~nm})$ trilayer stacks. As-deposited samples: Type $(\mathrm{A})-t_{(\mathrm{A})}{ }^{\mathrm{Tb}}=$ $100 \mathrm{~nm},\left(\mathrm{~A}^{*}\right)-t_{\left(A^{*}\right)}{ }^{T b}=50 \mathrm{~nm}$, and $(\mathrm{R})-t_{(R)}{ }^{T b}=5 \mathrm{~nm}$. Heated depositions: Type $(\mathrm{B})-t_{(B)}{ }^{T b}=100 \mathrm{~nm}$. (a) shows $\Theta-2 \Theta$ scans; and (b) surface-sensitive grazing incidence scans. Note, the sharp peak at $2 \Theta=33^{\circ}$ is substrate-related and comes from the $\mathrm{Si}$ beneath the $\mathrm{SiO}_{2}$ layer.

\section{FIGURE4:}

Simplified trilayer stack design as used for the estimation of the HCP* and HCP phase magnetisation $\left(M_{2}\right)$. This makes additional magnetometry of a pure FCC layer $\left(M_{1}\right)$ obsolete. The assumption of 
perfectly symmetric layers, sharing perfect interfaces, was necessary to allow for calculations only dependent on layer thickness $\left(t_{1}, t_{2}\right)$ rather than volume $\left(V_{l}, V_{2}\right)$.

\section{FIGURE 5:}

In-plane high-field magnetisation vs. temperature profiles of $\mathrm{Ta}(5 \mathrm{~nm}) / \mathrm{RE}(50 \mathrm{~nm}) / \mathrm{Ta}(5 \mathrm{~nm})$ trilayer stacks. The drop in magnetisation at $T=4 \mathrm{~K}$ compared to single-crystal saturation magnetisation is indicated as relative loss. (a) Thin films sputtered at elevated substrate temperature, whose maximal magnetisation was above the $\mathrm{Fe}_{65} \mathrm{Co}_{35}$ reference in the cryogenic regime. Threshold temperatures, where the $\mathrm{RE}$ magnetisation matches that of the $\mathrm{Fe}_{65} \mathrm{Co}_{35}$ reference, are indicated. (b) Thin films sputtered at room temperature, whose maximal magnetisation was below the $\mathrm{Fe}_{65} \mathrm{Co}_{35}$ reference.

\section{FIGURE 6:}

In-plane magnetometry of $\mathrm{SiO}_{2}: \mathrm{Ta}(5 \mathrm{~nm}) / \mathrm{Tb}(100 \mathrm{~nm}) / \mathrm{Ta}(5 \mathrm{~nm})$ trilayer stacks, Type (A) and (B). (a) Hysteresis loops recorded at $T=4 \mathrm{~K}$ to highlight different coercivities; and (b) zero-field-cooled-fieldcooled plots to indicate magnetic transitions.

\section{FIGURE 7:}

XRD $\Theta-2 \Theta$ and grazing incidence scans of (a) $\mathrm{SiO}_{2}: \mathrm{Ta}(5 \mathrm{~nm}) / \mathrm{DyGd}(50 \mathrm{~nm}) / \mathrm{Ta}(5 \mathrm{~nm})$; and (b) $\mathrm{Si}: \mathrm{Ta}(5 \mathrm{~nm}) / \mathrm{DyTb}(50 \mathrm{~nm}) / \mathrm{Ta}(5 \mathrm{~nm})$. Both alloys showed similar crystal growth comparable to pure RE metals, however the texture vanished towards the surface. Note, the sharp peak at $2 \Theta=33^{\circ}$ comes from $\mathrm{Si}$ and is substrate-related.

\section{FIGURE 8:}

In-plane magnetometry on DyGd and DyTb thin films. (a) and (b) high-field magnetisation vs. temperature profiles: (c) and (d) zero-field-cooled-field-cooled (ZFC-FC) plots recorded at field strengths of $\mu_{0} H=0.1 \mathrm{~T}$ and $\mu_{0} H=0.01 \mathrm{~T}$; (e) and (f) hysteresis loops recorded at $T=4 \mathrm{~K}$ with a maximum applied field of $\left|\mu_{0} H\right|=3 \mathrm{~T}$. 


\section{FIGURE 1:}

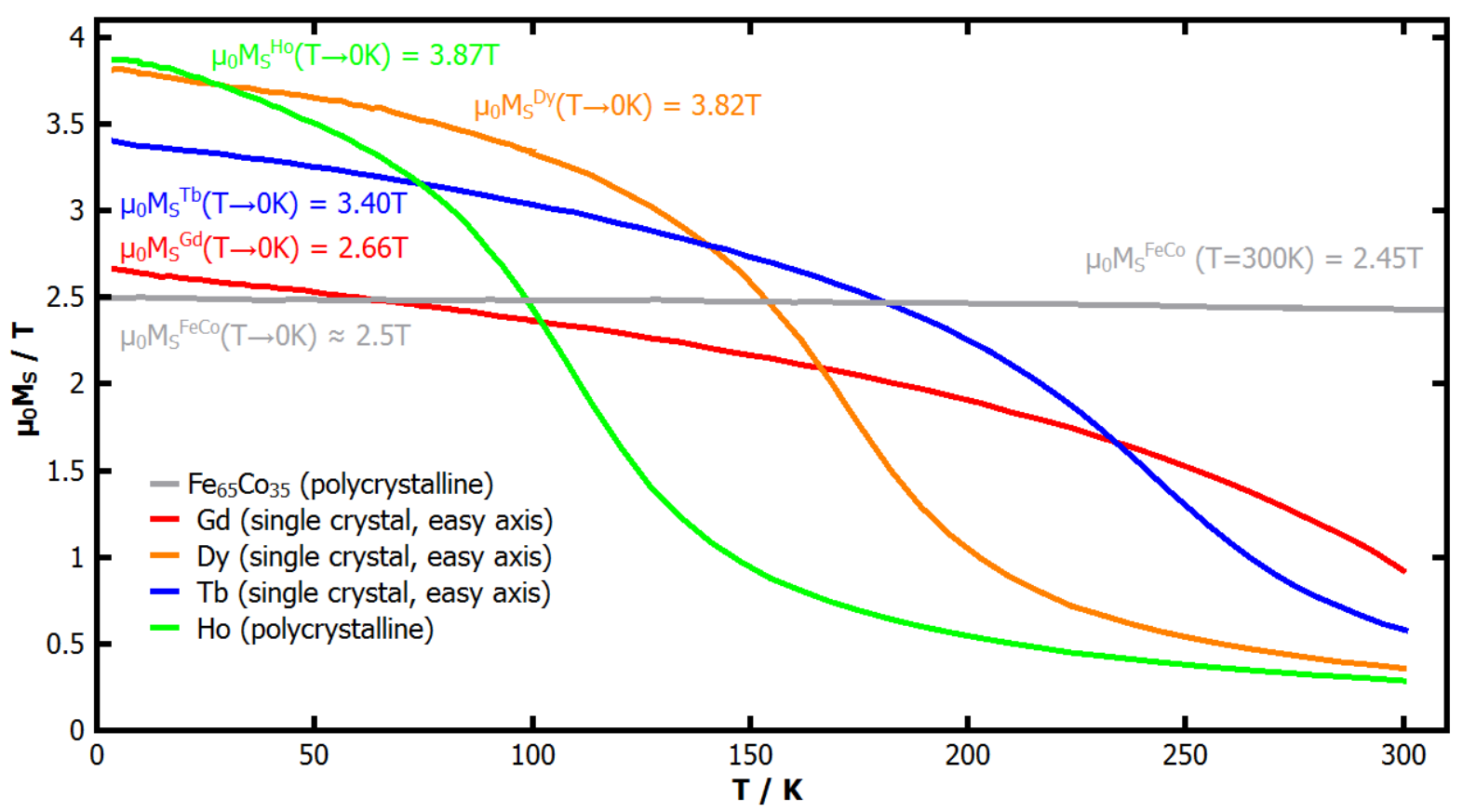


FIGURE 2:

(a)

room temperature

depositions

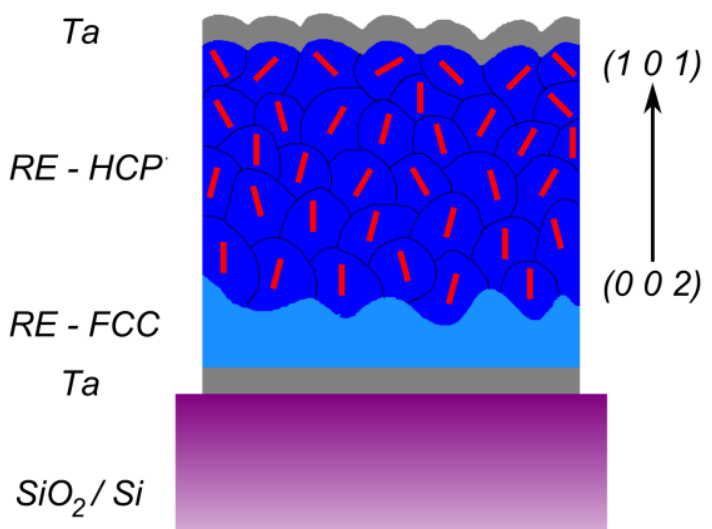

(b)

heated depositions

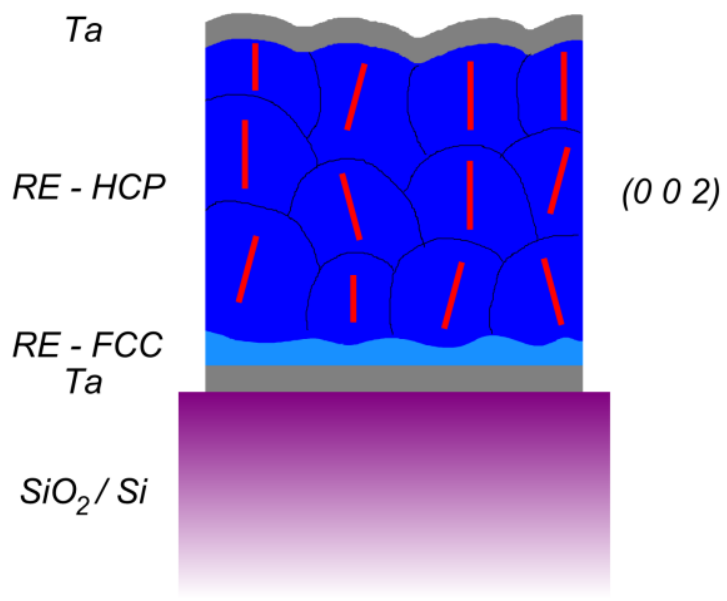


FIGURE 3:
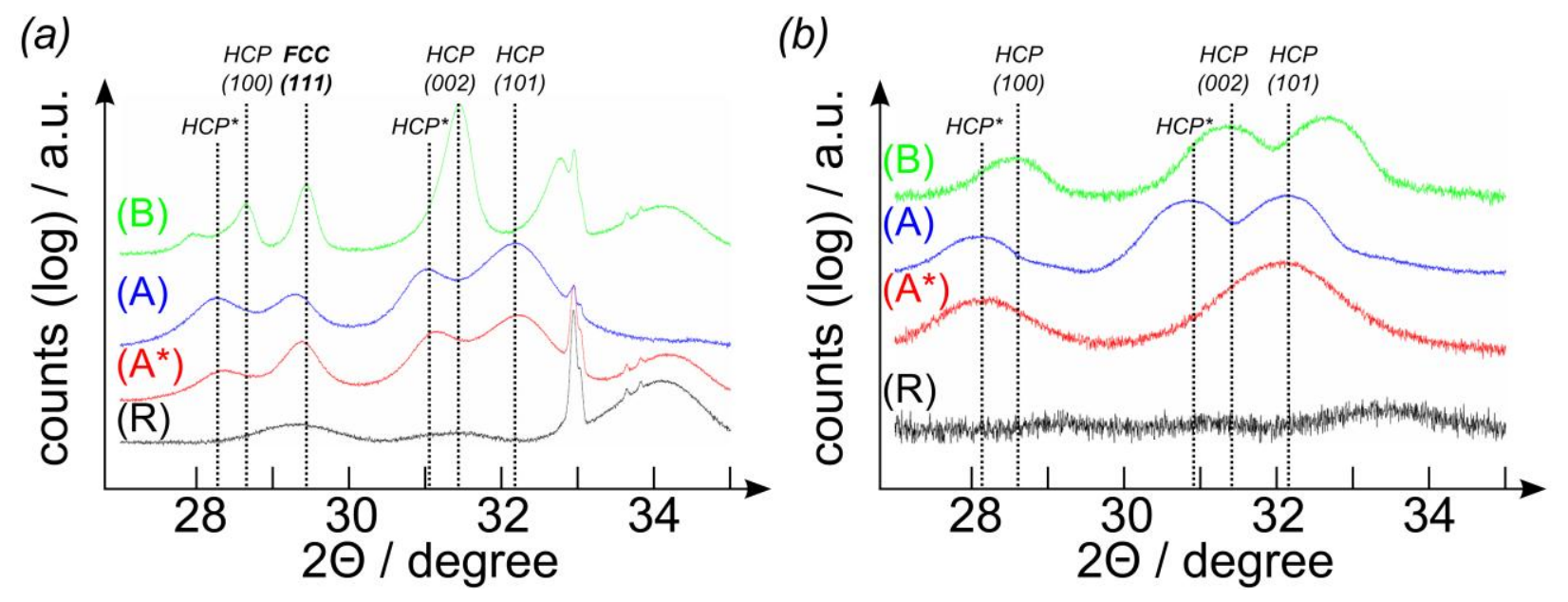
FIGURE 4:

(a)

room temperature

depositions

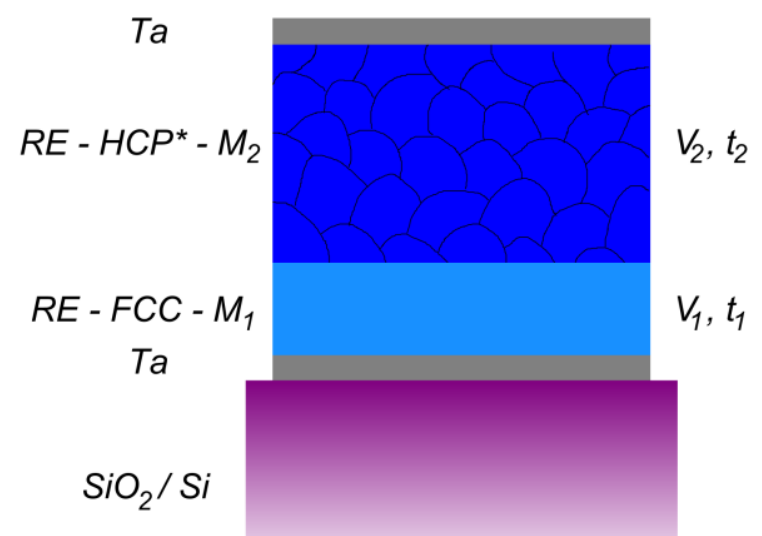

(b)

heated

depositions

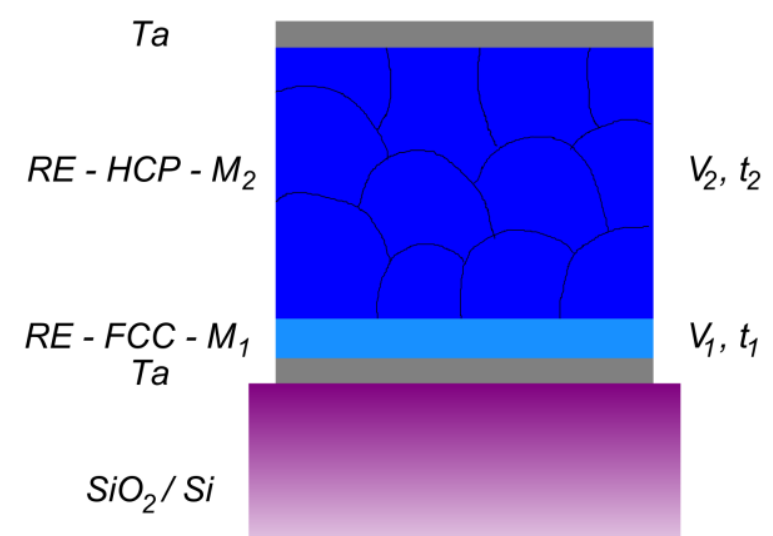


FIGURE 5:

(a)

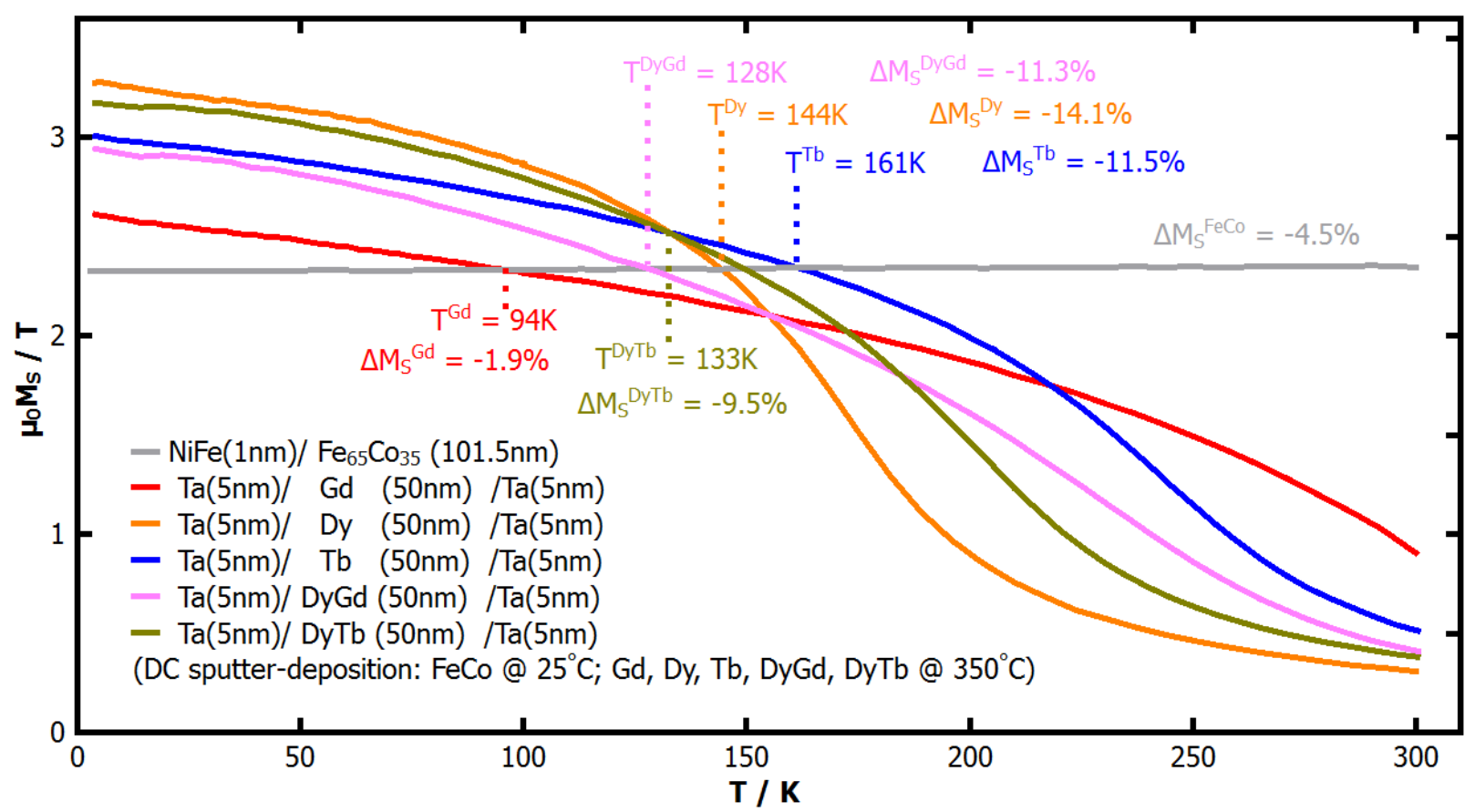

(b)

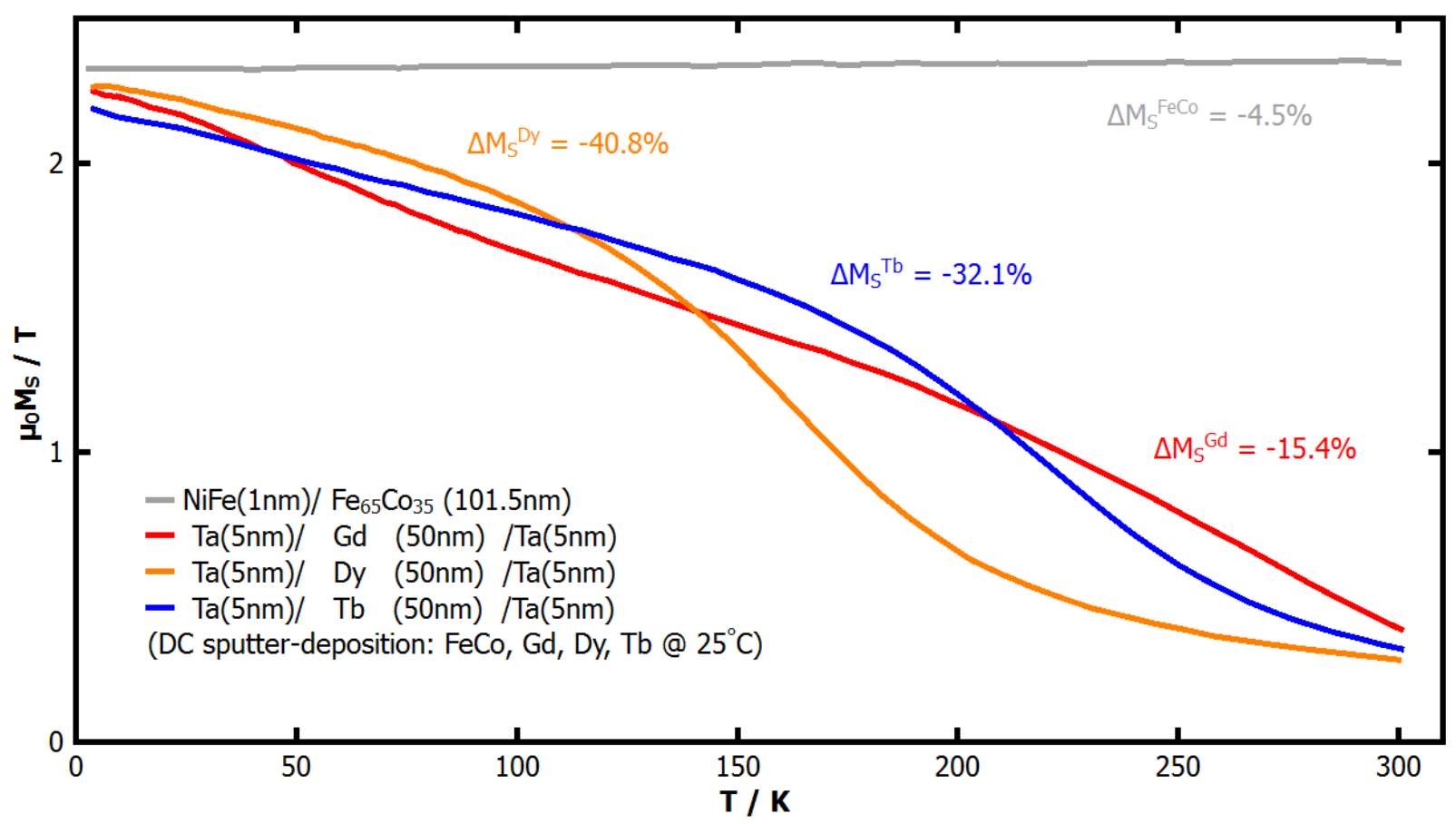


FIGURE 6:
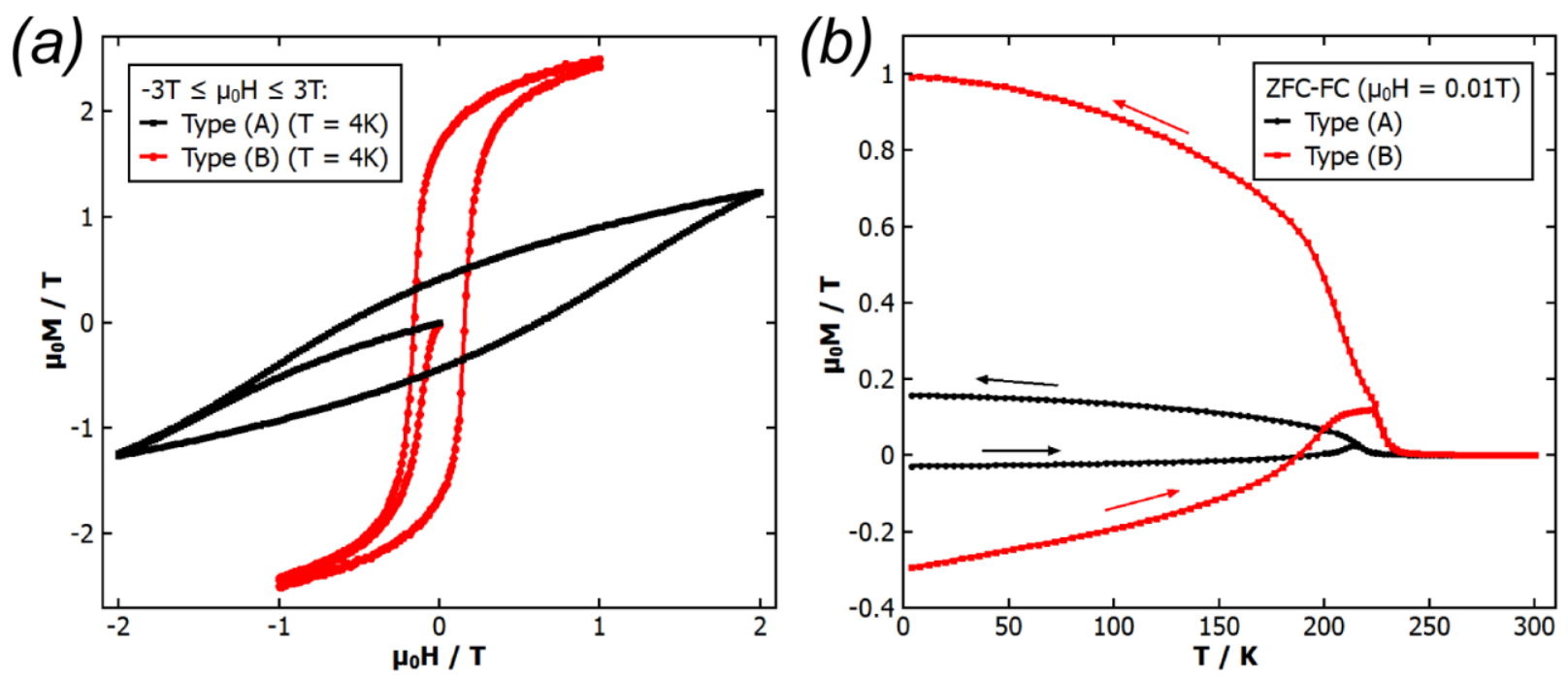
FIGURE 7:

(a)

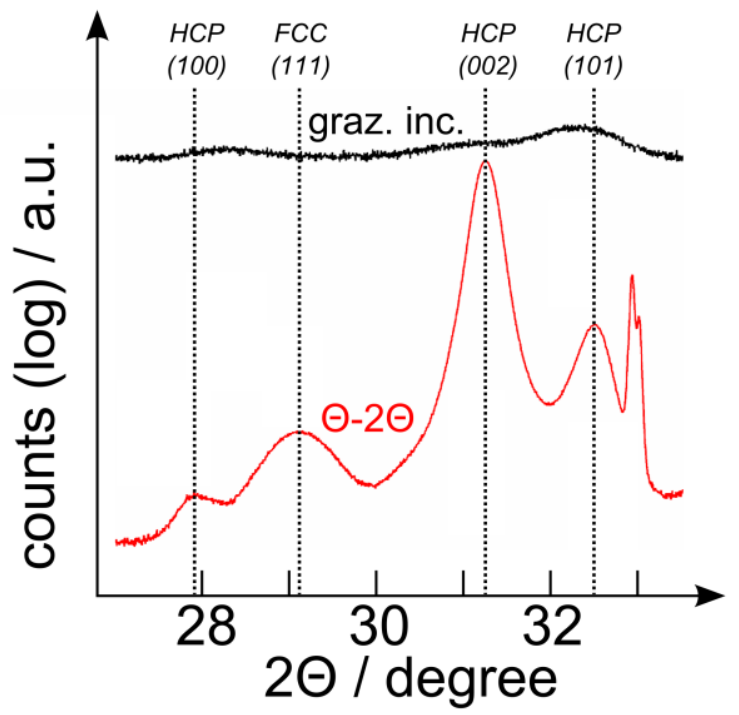

(b) $\quad$ DyTb

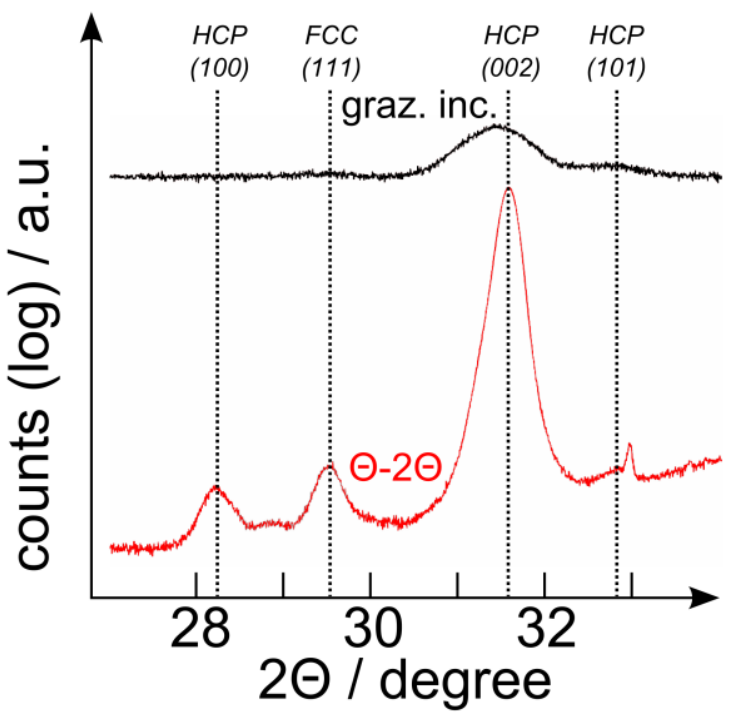


FIGURE 8:
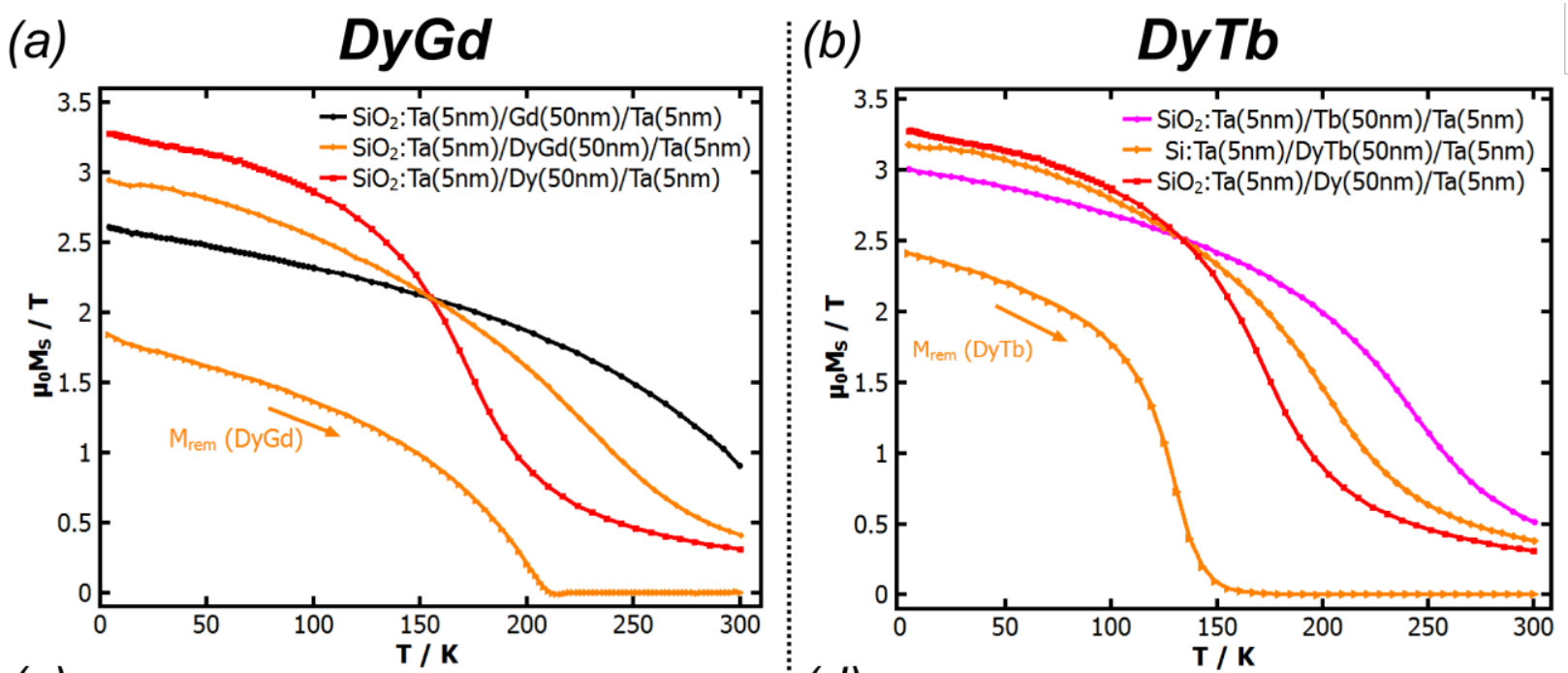

(c)

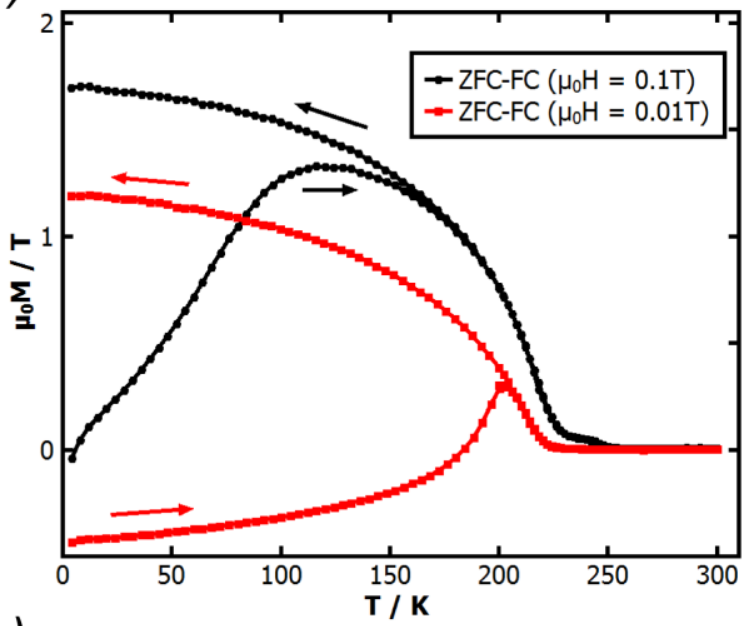

(e)

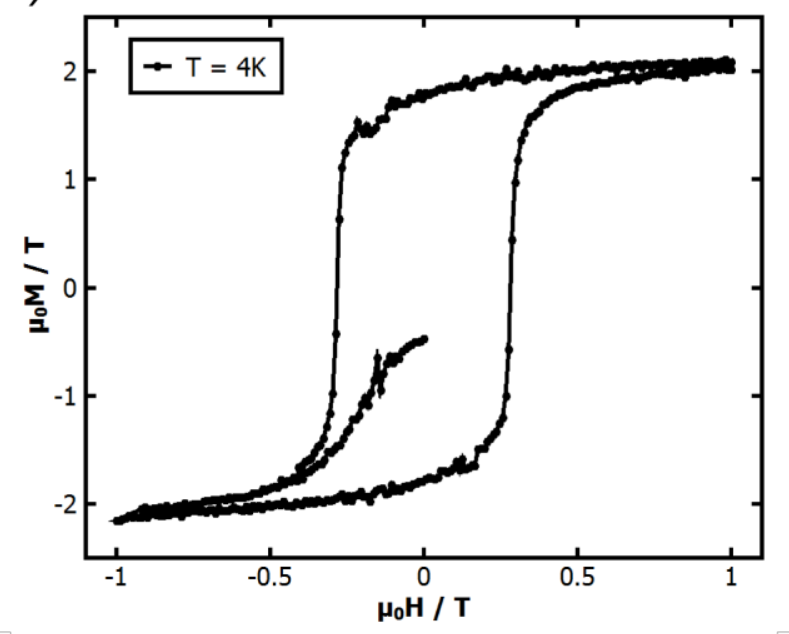

(d)

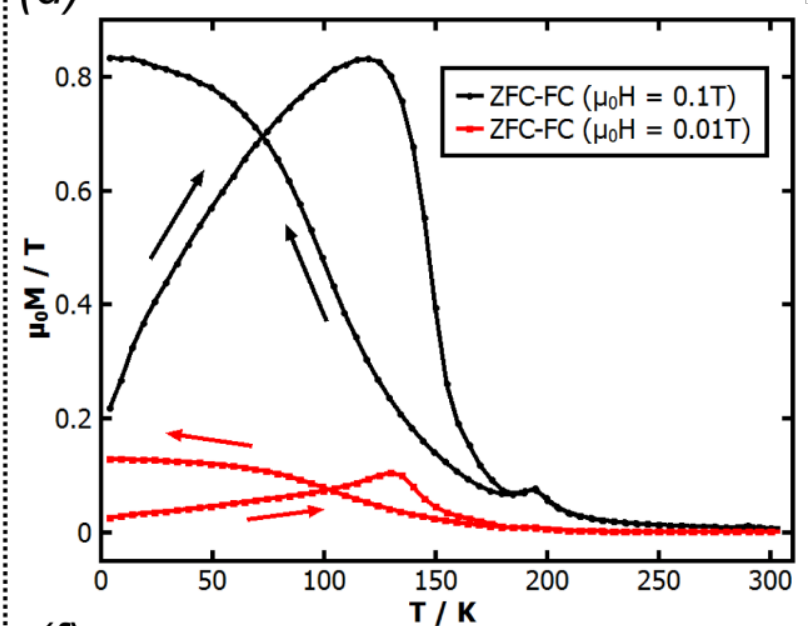

(f)

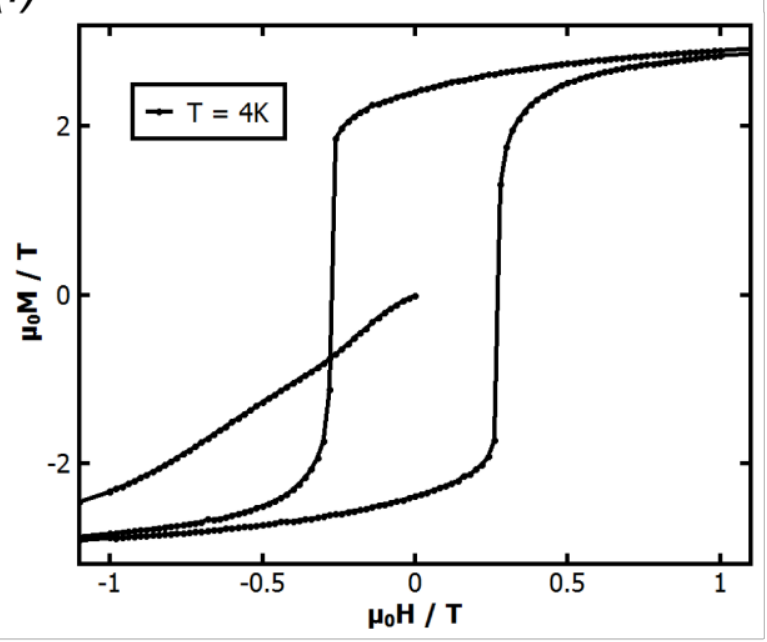

